\section{Leyendo los espacios: una aproximación crítica a la sintaxis espacial como herramienta de análisis arqueológico}

\section{Reading spaces: a critical approach to space syntax as archaeological analysis instrument}

Jesús Bermejo Tirado

Instituto de Historia, CCHS-CSIC. Madrid*

\section{Resumen}

Este trabajo pretende explicar de forma detallada los distintos elementos teóricos y metodológicos que conforman la sintaxis espacial. Se realiza una genealogía de sus bases teóricas como paso previo a la explicación de diversas herramientas analíticas. Éstas se ilustran con una serie de ejemplos históricos concretos. Se plantea un estudio comparativo con otras formas de análisis arquitectónico y arqueológico, del que se deduce una serie de problemas metodológicos. Por último se realiza una reflexión acerca de sus posibilidades interpretativas y se introducen algunas notas destinadas a plantear nuevas perspectivas de uso.

Palabras clave: Space syntax; entornos construidos; household archaeology; arqueología de la arquitectura; Juliobriga.

\section{Abstract \\ This work intends to explain in detail the different theoretical and methodological elements which belong to space syntax. A genealogy of its theoretical bases as preliminary step before the explanation of the different analytical instruments is firstly shown. A comparative study with other archaeological and architectural analysis is also offered, showing a group of methodological problems. The interpretative possibilities of space syntax analysis are settled in question and some final notes aimed to offer new perspectives are introduced.}

Keywords: Space syntax; built environments; household archaeology; archaeology of architecture; Juliobriga.

\footnotetext{
* jesus.bermejo@cchs.csic.es

${ }^{1}$ G. I. Mosaicos hispano-romanos: de lo artístico a lo social. Dirigido por la Dra. Guadalupe López Monteagudo (IH-CCHS) a quien quiero agradecerle el continuo apoyo y orientación que me proporciona. Quiero así mismo agradecer a todos mis compañeros del Instituto de Historia las discusiones teóricas metodológicas que periódicamente nos sirven para matizar, corregir y apuntalar diferentes ideas, algunas de las cuales se exponen en el presente trabajo. Especialmente agradezco a la Dra. M. a Ángeles Utrero (IH-CCHS) su estimulante discusión e interés para la elaboración de este trabajo. También agradezco las sugerencias planteadas por los árbitros anónimos de la revista y que han servido para revisar y explicitar algunos aspectos que mejoran la comprensión
} global del texto.
«Space creates special relations between function and social meaning».

B. Hillier y J.- Hanson (1984): The social logic of space: $1-2$

\section{INTRODUCCIÓN}

La tradición académica contemporánea tiende a considerar que la arquitectura está constituida fundamentalmente por dos elementos esenciales ${ }^{2}$ : construcción y espacialidad. Esta concepción del entorno construido hunde sus raíces en las vanguardias del siglo $\mathrm{XX}$, que en su vertiente arquitectónica propiciaron una reelaboración del discurso teórico, tomando al espacio como un elemento principal del diseño edilicio. La obra de grandes tratadistas como Frank Lloyd Wright, Mies Van de Rohe o Le Corbusier supuso, si no el descubrimiento del espacio en arquitectura, si la conciencia profunda de su importancia como marco de la vida humana ${ }^{3}$.

Esta nueva importancia del espacio arquitectónico se vio incrementada por diversas corrientes teóricas del siglo xx. Al hilo de la teoría contemporánea, el espacio dejó de ser una mera cualidad de la realidad física, como lo había concebido el paradigma cartesiano, para convertirse (además) en una construcción cultural. De este modo, arquitectura y urbanística no solamente tenían la capacidad de producir espacios, sino que también eran creadoras de ambientes o lugares 4 .

Antropólogos, sociólogos y filósofos de distinto cuño han desarrollado distintos modelos teóricos que han sido utilizados para analizar esos mismos valores sociales o culturales de los que estaban dotados los espacios arquitectónicos. Por una parte, algunos estructuralistas (y neoestructuralistas) como Lévi-Strauss ${ }^{5}$ y el Bourdieu etnógrafo ${ }^{6}$ desarrollaron un sistema cross-cultural de elementos semánticos aplicados a la espacialidad de las construcciones domésticas definidas como «estructuras estructuradotas». Otros autores, como H. Lefebvre ${ }^{7}$ o M. Foucault ${ }^{8}$, se han

2 Seguimos la definición de diversos autores: Zevi, B. (1951): Saber ver la arquitectura: ensayo sobre la interpretación espacial de la arquitectura. Trad. J. Bermejo Godoy. Ed. Poseidón. Buenos Aires: 5. Norberg-Schulz, C. (1963): Intentions in Architecture. Oslo. Id. (1988): Nightlands: nordic buildings. Mass. Cambridge: 8 .

${ }^{3}$ Calafell, E. (2000): Las unités d'habitacion de Le Corbusier: aspectos formales y constructivos. Fundación Caja de Arquitectos. Barcelona: 6-17. Gastón, C. (2005): Mies: el proyecto como revelación del lugar. Barcelona: 3-29.

${ }^{4}$ Richter, M. (1955): Espacio y ambiente en la arquitectura moderna. Gustavo Gili. Barcelona.

5 Lévi-Strauss, C. (1991): Voz «Maison». En Bonte, P. y Izard, M. (Eds.): Dictionnaire de l'ethnologie et de l'anthropologie. Presses Univiersitaires de France. Paris. 
encargado de dibujar, desde una perspectiva ciertamente más crítica y pesimista, la forma en la que los flujos ideológicos emitidos desde los centros de poder influyen de forma sutil y cotidiana en las configuración espacial del diseño arquitectónico moderno.

La arqueología histórica, fundamentalmente la de tradición continental, ha desarrollado diversos métodos analíticos con los que analizar la vertiente constructiva de la arquitectura desde un punto de vista histórico?. En paralelo a este desarrollo de instrumentos metodológicos, la arqueología protohistórica, sobre todo la emanada de los centros de investigación anglosajones, ha centrado en los últimos años su foco de atención en la vertiente espacial de la arquitectura ${ }^{10}$.

En este cuadro historiográfico, la inclusión de una serie de herramientas de análisis espacial, agrupadas bajo la ambigua denominación de space syntax, ha supuesto la apertura de una vía de estudio con la que interpretar determinados aspectos sociales e ideológicos, contenidos en el diseño y distribución de los espacios arquitectónicos ${ }^{11}$.

El desarrollo de este método de análisis espacial ${ }^{12}$ tiene un hito fundamental en el trabajo teórico desarrollado en la década de los ochenta por Bill Hillier y una serie de alumnos con sede en The Bartlett ${ }^{13}$ (Londres), un centro de investigación multidisciplinar destinado al estudio de los ambientes construidos. Desde entonces la sintaxis espacial ha sido aplicada por diversos investigadores centrados en materias tales como urbanismo, salud pública, sociología, antropología o ingeniería civil, y actualmente se ha convertido en un auténtico foco de actividad interdisciplinar ${ }^{14}$.

\footnotetext{
${ }^{6}$ Bourdieu, P. (1973): «The Berber house». En Douglas, M. (Ed.): Rules and meaning. Penguin. Suffolk: 98-110.

${ }^{7}$ Lefebvre, H. (1991): The Production of Space. University Press. Cambridge.

${ }^{8}$ Foucault, M. (1980): «El ojo del poder». En Bentham, J: El Panóptico. Ed. La Piqueta. Madrid: iii-xi.

${ }^{9}$ Caballero Zoreda, L. y Escribano Velasco, C.(1996): «El método arqueológico aplicado al proceso de estudio e intervención en edificios históricos». En Arqueología de la Arquitectura. Junta de Castilla y León. Burgos.

10 Ayán Vila, X. M.; Blanco Rotea, R. y Mañana Borrazás, P. (2003): "Archaeotecture: seeking a new archaeological vision of architecture». En Ayán Vila, X. et alii (Eds.): Archaeotecture: Archaeology of Architecture. BAR International Series 1175. Archaeopress. Oxford: 1-15.

${ }^{11}$ Hillier, B.; Hanson, J.; Graham, H. (1987): «Ideas are in things: an application of space syntax method to discovering house genotypes». Environment and Planning B: Planning and Design. 14: 363-385.

${ }^{12}$ Hillier, B. y Hanson, J. (1984): The Social Logic of Space. University Press. Cambridge. En adelante SLS.

${ }^{13}$ Faculty of Built Enviorment (UCL). http://www.bartlett.ucl.ac.uk/index.php

${ }^{14}$ Las referencias bibliográficas son insondables. Un repositorio de acceso para investigadores en http://eprints.ucl.ac.uk/view/subjects/14500.html donde se pueden consultar 196 publicaciones científicas.

También hemos de reseñar la celebración de reuniones científicas bienales (International Space Syntax Symposium) de los que se han celebrado siete
} ediciones hasta la fecha.
La arqueología no ha sido una excepción, desde hace algunos años se vienen publicando sucesivos estudios sobre la aplicación de herramientas analíticas procedentes de la sintaxis espacial. En el marco de la arqueología anglosajona, la introducción de esta clase de análisis se ha convertido en algo relativamente frecuente ${ }^{15}$. Su introducción en el ámbito de la arqueología iberoamericana ha sido más tímida y tangencial, centrada sobre todo en el ámbito de la protohistoria de la Península Ibérica ${ }^{16}$ y en la arqueología histórica latinoamericana ${ }^{17}$. Es por ello que consideramos adecuada la realización de una síntesis crítica destinada a discutir las posibilidades y limitaciones de su aplicación para el análisis de realidades arquitectónicas del pasado histórico. El presente texto pretende realizar una caracterización de aquellos instrumentos analíticos que se han englobado bajo está etiqueta, contextualizando sus raíces teóricas y definiendo nuevas aplicaciones concretas para los mismos.

\section{LAS BASES TEÓRICAS: ANTROPOLOGÍA ESTRUCTURAL Y BUILT ENVIRONMENT STUDIES}

Antes de proceder a explicar las herramientas de análisis desarrolladas por la sintaxis espacial, creemos conveniente realizar una genealogía de los cimientos conceptuales sobre las que se ha apoyado este método.

La propia denominación de space syntax nos remite a la analogía textual como instrumento de análisis cultural. Este modelo hermenéutico tiene un referente fundamental

\footnotetext{
${ }^{15}$ Hemos de destacar la especial aceptación que ha tenido en la arqueología clásica. Laurence, R. (1994): Roman Pompeii: space and society. Routledge. Londres. Grahame, M. (2000): Reading Space: Social Interaction and Identity in the Houses of Roman Pompeii. BAR Int. Ser. 886. Archaeopress. Oxford. Id. (1999): "Reading the roman house: the social intepretation of spacial order». Proceedings of III TRAC Conference. Cruitnhe Press. Glasgow: 48-74. Kaiser, A. (2001): The Urban Dialogue: An analysis of the use of space in the. Roman city of Empúries, Spain. BAR Int. Series 901. Archaeopress. Oxford. Westgate, R. (2007): «House and society in Classical and Hellenistic Crete: a case study in regional variation». AJA 111: 423-57.

16 Fumadó, I. (2007): "Introducción al estudio de los baños domésticos de tradición fenicio-púnica». Saguntum. 39: 103-115. Jiménez Ávila, J. (2005): "Cancho Roano: el proceso de privatización de un espacio ideológico». TP. 62 (2): 105-124. Ayán Vila, X. M. (2003): «Arquitectura como tecnología de construcción social». Arqueología de la Arquitectura. 2: 17-24. Sánchez, J. (1998): «La arqueología de la Arquitectura. Aplicación de nuevos modelos de analítica a estructuras de la Alta Andalucía en época ibérica». TP. 55 (2): 89-109

17 Zarankin, A. y Niro, C. (2007): «La materialización del sadismo. Arqueología de la arquitectura de los centros clandestinos de detención de la Dictadura militar argentina (1976-1983)». En Funari, P. P. y Zarankin, A. (Eds.): Arqueología de la represión y la resistencia en América Latina, 1960 1980. Facultad de Filosofía y Humanidades. Univerisdad de Catamarca: 159182. Funari, P. P. y Zarankin, A. (2003): «Social archaeology of housing from a Latin American perspective: A case study». Journal of Social Archaeology. 3: $23-45$.
} 
en los trabajos de F. de Saussure ${ }^{18}$, quien a principios del siglo XX dejó sentadas las bases de la teoría lingüística y semiótica contemporánea por medio de la definición del concepto de signo, es decir, la asociación de una imagen o icono (significante) y un concepto (significado).

La capacidad semántica de los signos lingüísticos estaba condicionada, según Saussare ${ }^{19}$, por las relaciones que le unían a otros signos de una lengua, de manera que no es posible aprenderlos sin contextualizarlos en una red de imbricaciones. El lenguaje, pues, debía presentarse como una organización (una estructura), ya que los elementos lingüísticos no tienen ninguna realidad independiente de su relación con el todo.

Esta noción del lenguaje como estructura contextual de significados, fue asumida por C. Lévi-Strauss ${ }^{20}$ como referente básico de su antropología estructural ${ }^{21}$. Según el marco teórico planteado por el autor, dentro de una cultura, el significado es producido y reproducido a través de varias prácticas, fenómenos y actividades como sistemas de significación. El desarrollo del estructuralismo tuvo en la antropología su principal área de aplicación, dando lugar a la búsqueda de estructuras de significado por medio del estudio de los diversos elementos culturales (parentesco, religión, cultura material, etc.).

La cultura material era concebida por los estructuralistas como elementos dotados de un significado legible en un determinado contexto cultural. Desde esta perspectiva, algunos antropólogos (y prehistoriadotes como A. LeroiGourham $^{22}$ ) se lanzaron a una búsqueda de comparaciones trans-culturales con el objetivo final de reconstruir las leyes que, del mismo modo que en la lingüística, regían la configuración cultural de los grupos humanos.

Dentro de esta búsqueda de sistemas de significados culturales, una gran parte del trabajo del antropólogo A. Rapoport se centró en el espacio arquitectónico como elemento dotado de elementos semánticos ${ }^{23}$. A lo largo de sus trabajos propone un panorama en el que los elementos arquitectónicos pueden ser interpretados como formas dotadas de significados culturales, los cuales dividía entre aque-

${ }^{18}$ Saussare, F. (1964 [1916]): Curso de lingüística general. Ed. Losada. Buenos Aires.

${ }^{19}$ Saussare, F. (1964 [1916]): op cit. 99-107.

${ }^{20}$ Lévi-Strauss, C. (2000 [1961]): Antropología estructural. Paidós. Barcelona.

${ }^{21}$ Henaff, M. (1991): Claude Lévi-Strauss et l'anthropologie structurale. Belfond. Paris: $13-45$.

${ }^{22}$ Leroi-Gourham, A. (1984). Simbolos, Artes y Creencias de la Prehistoria. Istmo. Madrid.

${ }^{23}$ Hacemos referencia de forma general a dos de sus obras más conocidas. Rapoport, A. (1969): House form and culture. Englewood Cliffs. Prentice-Hall. New Jersey. Id. (1982): The meaning of the built environment: a non-verbal communication approach. Sage Publications. Beverly Hills.

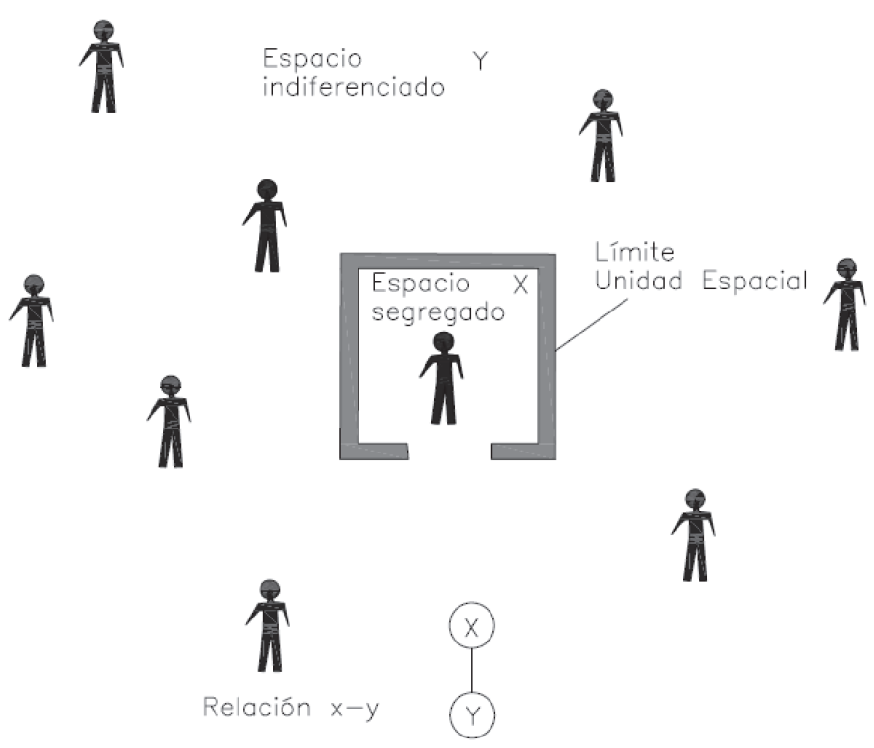

Fig. 1. Gráfico en el que se expresa el efecto del límite espacial y la accesibilidad del individuo inscrito en él para mantener un encuentro social. El individuo inscrito en el espacio $\mathrm{X}$ está sometido a la restricción en las posibilidades de mantener un encuentro social, respecto a los individuos situados en el espacio $Y$

llos que ofrecen un significado verbal (es decir, aquellos que poseen un referente semántico de tipo metafórico; p. e. en la domus romana tardorrepublicana, el atrium era el espacio donde el paterfamilias recibía a los clientes como símbolo de su prestigio social, era pues metáfora de la magnificentia del patrón) y otros dotados de significados no-verbales (aquellos que no tienen un referente metafórico expresable en un concepto lingüístico; p. e. en la domus romana, los siervos y clientes solo podían acceder al vestibulum o al atrium, mientras que los invitados más selectos podían pasar sin problemas a partes más interiores de la casa). De esta forma, siguiendo esta forma de interpretación, muchos de estos significados no-verbales servían para estructurar las relaciones sociales dentro de un complejo arquitectónico.

La complejización de las estructuras sociales suele acompañarse de sistemas de significados variados, que igualmente necesitan un desarrollo arquitectónico-espacial lo bastante complejo como para poder actuar como expresión de estos significados verbales y no-verbales. Esta apreciación de los estudios arquitectónicos y urbanísticos como materialización de significados sociales y culturales ha dado lugar al surgimiento de los llamados EBS (Environment Behavior Studies) $)^{24}$, es decir, una perspectiva analítica en la que se tratan de documentar las formas en que la configuración espacial de los entornos construidos se relacionan con la conducta o los principios culturales de los seres humanos.

\footnotetext{
${ }^{24}$ Rapoport, A. (1990): «Systems of activities and systems of settings». En Kent, S. (Ed.): Domestic architecture and the use of space: An interdisciplinary crosscultural study. NDA. University Press. Cambridge: 9-20.
} 


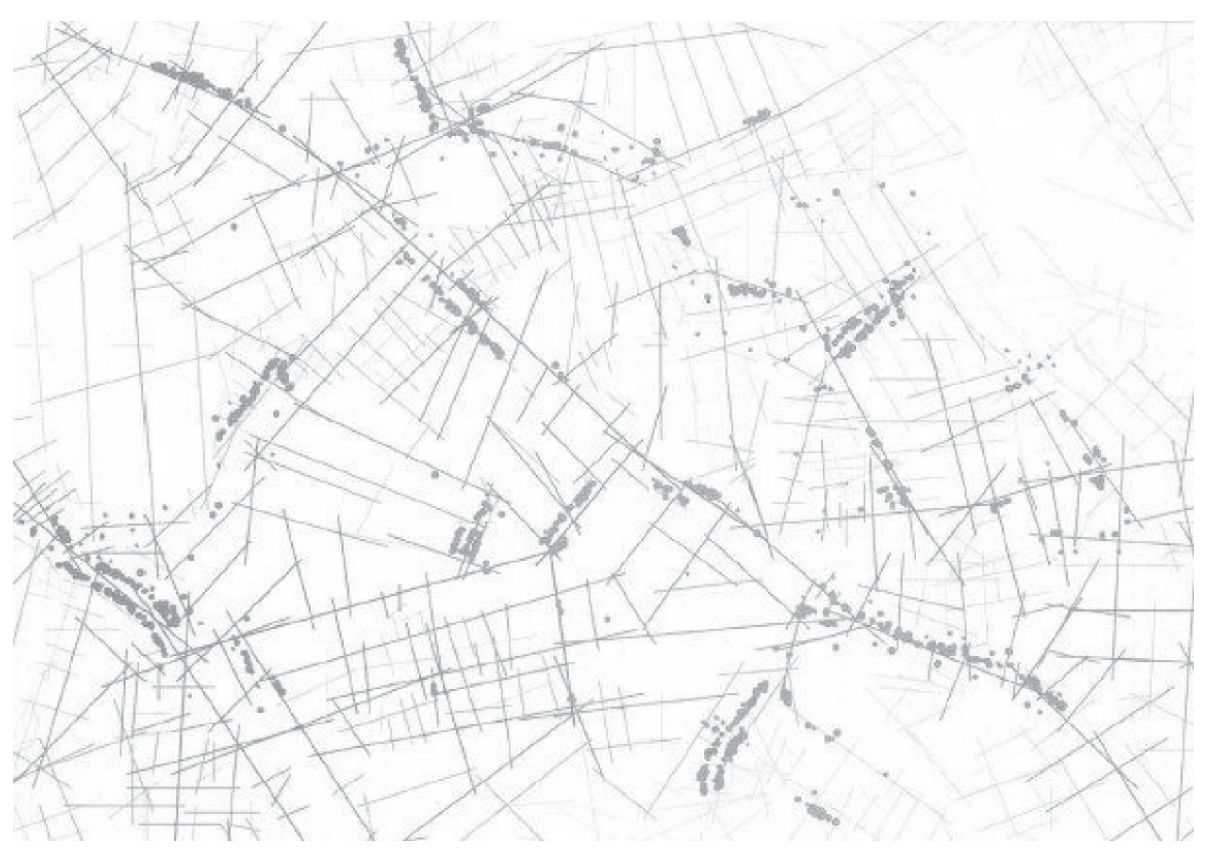

Fig. 2. Gráfico de axialidad de Candem Town (Londres) a partir de B. Hillier (1999): Fig. 7. Las líneas de mayor grosor representan las áreas de mayor convergencia en el trazado urbano del mercado. Se puede apreciar cómo las grandes concentraciones comerciales (representadas como grupos de puntos) coinciden a grandes rasgos con las líneas de mayor convergencia ${ }^{25}$
Con este bagaje, la sintaxis espacial surge como una vía para el estudio de estos significados no-verbales (o no-discursivos, según la terminología aplicada por B. $\operatorname{Hillier}^{26}$ ), que tiene especiales posibilidades de aplicación para registrar la configuración espacial como elemento articulador de las relaciones sociales de los grupos humanos que las crean. Del mismo modo que la sintaxis lingüística estudia las relaciones de ordenamiento y jerarquía entre los distintos miembros de una oración (sin entrar en su contenido semántico concreto), la space syntax estudia las formas en las que se vinculan y organizan los espacios de un conjunto arquitectónico, tratando de inferir aquellos aspectos de la estructuración social que pudieron influir en su diseño. A través de este método podemos, por ejemplo, registrar el grado de jerarquización que opera en la configuración espacial de un ámbito concreto, pero no el tipo de estructura social que habitó ese mismo espacio. En palabras de B. Hillier y J. Hanson $^{27}$ : "The fundamental proposition of the syntax theory is not that there is a relation between settlement for$\mathrm{ms}$ and social forces, but that there a relations between the generators of settlement forms and social forces».

\section{HERRAMIENTAS ANALÍTICAS DE LA SINTAXIS ESPACIAL: ALGUNOS CONCEPTOS FUNDAMENTALES}

La publicación de The social logic of space servirá como catalizador de una serie de técnicas de análisis que nos

${ }^{25}$ Hillier, B. (1999): "Centrality as a process: accounting for attraction inequalities in deformed grids». Urban design. 4 (3-4): 107-127.

${ }^{26}$ Hillier, B. (1996): Space is the machine. University Press. Cambridge: 305-306.

${ }^{27}$ SLS, op. cit. supra n. 12: 82-83. proporcionan herramientas a través de las que registrar (expresados gráficamente por un lado y cuantificados de forma numérica por otro) la estructuración espacial de los entornos arquitectónicos y los significados no-discursivos que de ellos se pueden derivar.

Su texto parte de la conceptuación de la célula espacial como unidad fundamental de análisis. La explicación de la célula o unidad espacial se deriva del concepto de límite como elemento separador, destinado a definir una región o espacio y segregarlo de aquello que podemos denominar como «espacio indiferenciado» ${ }^{28}$. Esta segregación es aquello que posibilita un encuentro social, con un grado diverso de accesibilidad, respecto al espacio indiferenciado o a otras unidades espaciales. El límite que define la unidad espacial crea una «discontinuidad fundamental $»^{29}$ que se relaciona con el resto de la organización espacial a través de la permeabilidad. Aunque pueda parecer una contradicción, un límite espacial sólo es socialmente inteligible ${ }^{30}$ si mantiene una relación de permeabilidad con otras unidades espaciales o con el espacio indiferenciado.

El grado de presencia ${ }^{31}$, o accesibilidad para un encuentro social, de una unidad espacial dependerá por lo tanto del número y naturaleza de las relaciones de per-

${ }^{28}$ SLS, op.cit.supra n. 12: 73 ss.

${ }^{29}$ SLS, op. cit. supra n. 12: 144.

${ }^{30} \mathrm{La}$ abstracción de un espacio sin relación de permeabilidad con otras entidades espaciales, aunque posible desde un punto de vista teórico, no tiene interés alguno desde el punto de vista del análisis social.

${ }^{31}$ Grahame, M. (1997): "Reading the roman house: the social interpretation of spatial order». En Proceedengs of the III TRAC Conference. Cruithine Press. Glasgow: $49-74$. 


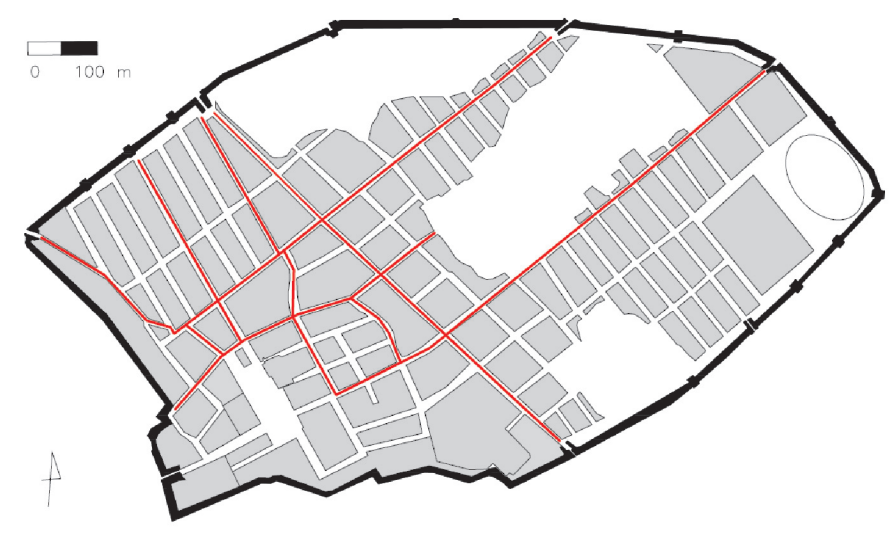

Fig. 3. Plano esquemático de Pompeya con principales líneas de convergencia axial. A partir de Laurence (1994)

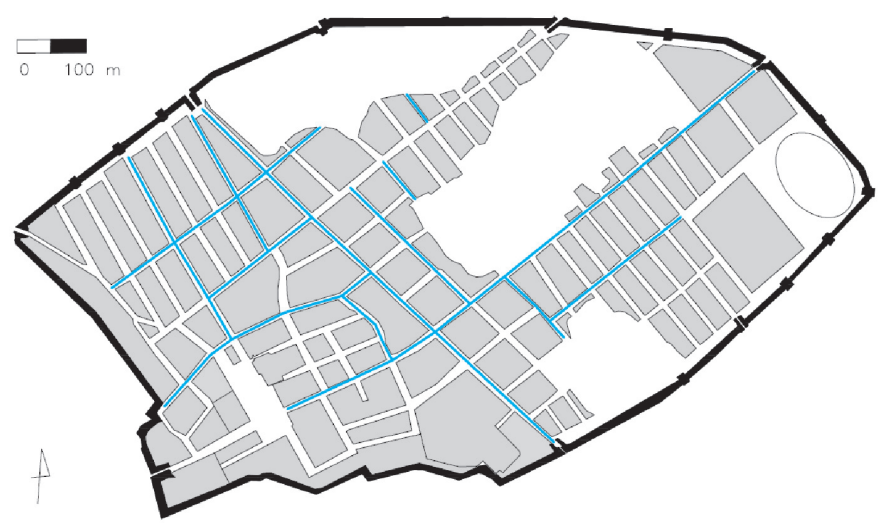

Fig. 4. Plano esquemático de Pompeya con las principales áreas de concentración de graffiti en las fachadas. A partir de Laurence (1994)

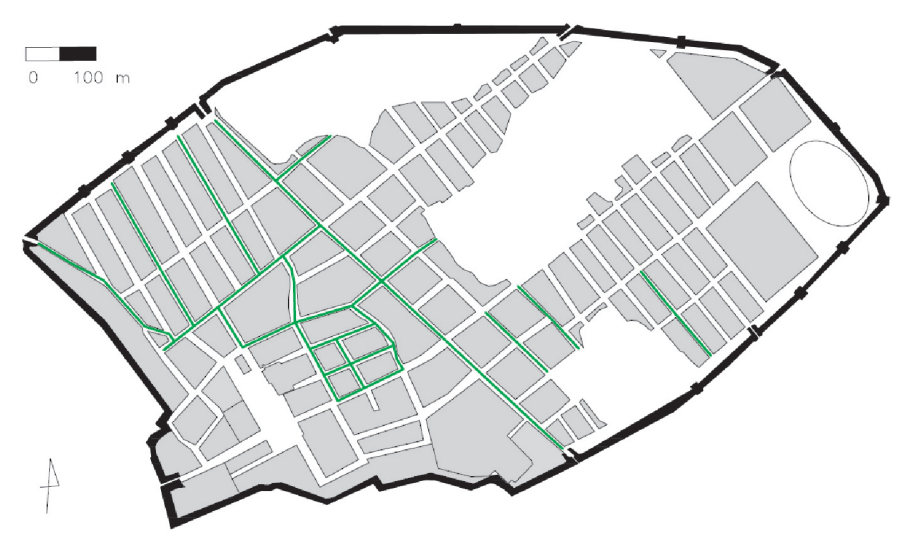

Fig. 5. Plano esquemático de Pompeya con la mayor concentración de áreas de servicio/tabernae de la ciudad. A partir de Laurence (1994)

meabilidad respecto a otras unidades espaciales. Esto implica que el espacio construido tiene un significado social en función de su orden relacional. Este orden crea y reproduce un modelo particular de permeabilidad caracterizado por la yuxtaposición de espacios con diferentes niveles de presencia o accesibilidad.

\section{Herramientas de representación gráfica}

A partir del establecimiento de las diversas unidades espaciales de un complejo arquitectónico o urbanístico, la sintaxis espacial plantea una serie de elementos de representación gráfica destinados a ilustrar diversas características de la lógica social que estructura los entornos construidos, complementando así las tradicionales planimetrías bidimensionales. A continuación vamos a tratar de explicar cuáles son los principales métodos de representación gráfica desarrollados por la sintaxis espacial ${ }^{32}$.

\section{Mapas de convergencia}

Se trata de una de las herramientas básicas para la visualización del genotipo o dinámica social que sirven para ayudar a reconstruir el desarrollo de un entorno construido. Se trata de establecer una serie de líneas axiales ${ }^{33}$, surgidas del análisis de cada edificio, cuya prolongación en línea recta se amplía hasta el siguiente edificio. Las áreas de convergencia, surgidas de la intersección de esas líneas de axialidad, nos sirven para ilustrar los principales nodos de reunión social dentro de un complejo urbanístico o asentamiento. Estos mapas se pueden utilizar para la recreación de áreas de actividad social o económica dentro de un asentamiento (Fig. 2, 3, 4, 5). Asimismo se han utilizado para decidir qué áreas son las más idóneas para el emplazamiento de determinados equipamientos urbanísticos.

\section{Gráficos de accesibilidad}

Los gráficos de accesibilidad (gamma analysis) han sido definidos como un método topográfico que nos permite la representación y la interpretación de las configuraciones espaciales en edificios y asentamientos ${ }^{34}$. Estos gráficos Gamma, como los han bautizado B. Hillier o J. Hanson, son en realidad la adaptación de los gráficos de axialidad para la comprensión de la presencia/permeabilidad de las unidades espaciales ${ }^{35}$. Estos mapas de accesibilidad están fundamentalmente orientados al análisis interior de edificios.

\footnotetext{
32 SLS, op. cit. supra n. 12: 90-104. Widlok, T. (1999): «Mapping Spatial and Social Permeability». Current Anthropology. 21 (3): 392-400.

${ }^{33}$ Para una explicación gráfica de la formación de líneas de axialidad vid. SLS: Figs. 26, 27, 28.

${ }^{34}$ Hillier, B.; Hanson, J. y Graham, H. (1987): «Ideas are in things: an application of space syntax method to discovering house genotypes». Environment and Planning B: Planning and Design. 14: 363.

${ }^{35}$ SLS, op. cit. supra n. 12: 147.
} 

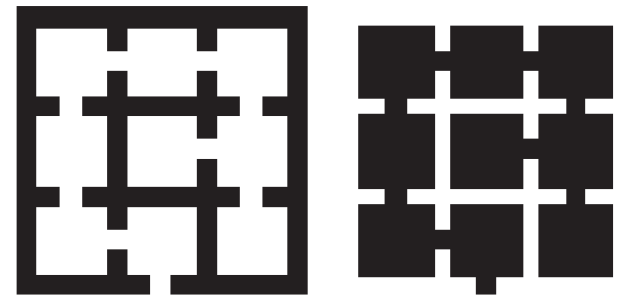

a.
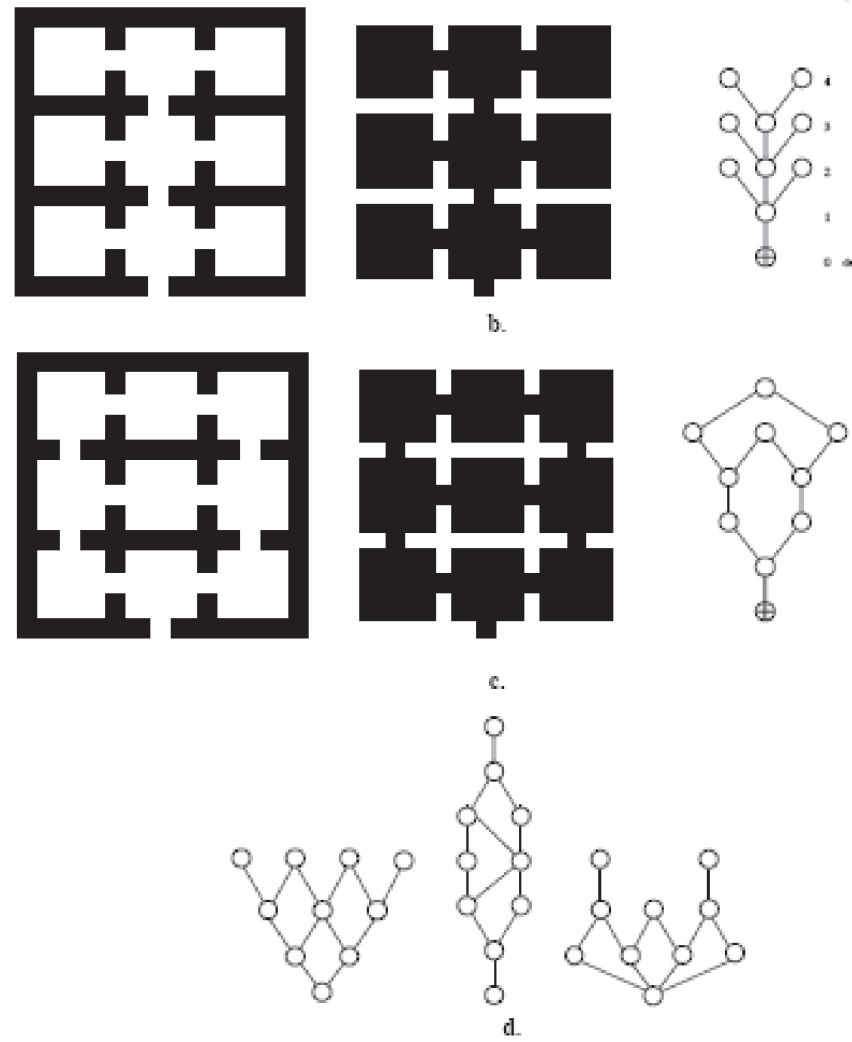

Fig. 6. Tres planimetrías, aparentemente similares, con sus tres gráficos de accesibilidad completamente diferentes. Cada uno de ellos representa un modelo de permeabilidad/presencia distinto. Según Hillier (1996)

Para ello se representa cada unidad espacial por medio de un círculo. Los «espacios de transición» o conexiones ${ }^{36}$ entre unidades espaciales se representan por medio de líneas que nos indican las relaciones de accesibilidad entre ellas.

Una vez hecho esto, y para dotar de coherencia al conjunto, habremos de «justificar» el gráfico, es decir, reordenarlo en relación al espacio exterior ${ }^{37}$. Este punto de referencia se sitúa en la base del gráfico justificado, es la representación del espacio que contiene todo el gráfico y por lo tanto sirve como inicio de la ordenación con respecto a su accesibilidad (Fig. 6).

\footnotetext{
${ }^{36}$ SLS, op. cit. supra n. 12: 45.

${ }^{37}$ Dicho espacio exterior no tiene valor como unidad espacial y suele representarse como un círculo con una (+) en su interior.
}

\section{Gráficos de Visibilidad}

Este tipo de gráfico se basa en el concepto de isovista ${ }^{38}$, que consiste en el área de un entorno construido directamente visible desde una localización dentro de un espacio (un punto generador). La representación de esta área en un entorno construido suele generar un polígono referenciado desde el punto generador (Fig. 9).

La formulación geométrica de la isovista, del polígono que genera, fue concebida como un índice fundamental para medir relaciones espaciales a través de la exploración de estos campos de visibilidad. Sin embargo, el desarrollo primario de este concepto analítico no estuvo acompañado de una propuesta de aplicación de la misma al análisis social $^{39}$.

La propuesta de utilización de la isovista desde un punto de vista interpretativo se produjo años más tarde en el contexto del desarrollo metodológico provocado por la space syntax. De esta forma, un grupo de teóricos de la arquitectura ${ }^{40}$ desarrolló el concepto de gráfico de visibilidad como una forma de representación complementaria a las expuestas por Hillier y Hanson.

Demostrando el poco valor analítico que tenían las isovistas de forma individualizada, pues no tienen en cuenta las variaciones de visibilidad entre puntos internos, crearon una metodología para representar la relación visual de una serie de puntos generadores dentro de un entorno construido. Su método, denominado gráfico de visibilidad, sirve para representar distintos tipos de relación visual dentro de espacios construidos. Para trazar uno de estos gráficos, previamente se ha de trazar una rejilla de puntos generadores sobre un plano. Estos puntos se han de establecer teniendo en cuenta algún criterio analítico. Una buena solución para intentar adaptar una escala visual humana es establecer una rejilla de puntos generadores situados de manera equidistante a un metro unos de otros.

Una vez tengamos estos vértices, podemos establecer relaciones de visibilidad entre sucesivos ejes dispuestos dentro de un determinado ambiente construido. Así estas relaciones pueden ser de dos tipos: primarias, cuando los dos vértices de un eje están contenidos dentro de la misma

\footnotetext{
${ }^{38}$ Benedikt, M. L. (1979): "To take hold of space: isovists and isovist fields». Environment and Planning B. 6: 47-65.

${ }^{39}$ Benedikt, M. L. y Burnham, C. A. (1985): «Perceiving architectural space: from optic rays to isovists". En Warren, W. H. y Shaw, R. E. (Eds.): Persistence and Change. Londres: 34-48.

40 Turner, A.; Doxa, M.; O’Sullivan, D. y Penn, A. (2001): «From isovists to visibility graphs: a methodology for the analysis of architectural space». Environment and Planning B. 28: 103-21.
} 
Fig. 7. Planimetría esquemática de la Domus 2 de la Llanuca (Juliobriga) a partir de P.A. Fernández Vega 1993, con propuesta de cierre del muro $\mathrm{O}$ en rojo. Gráfico de accesibilidad sin justificar

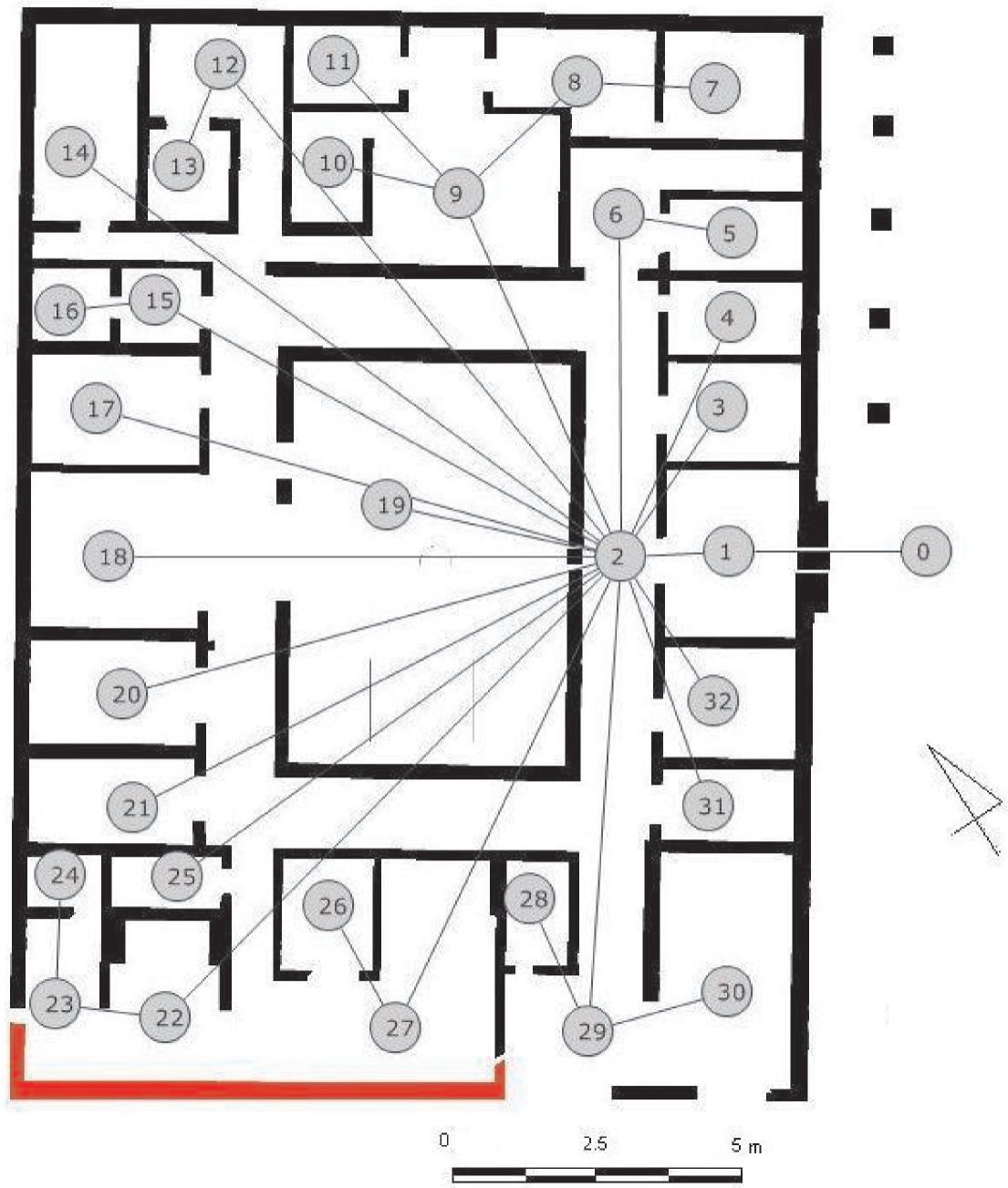

isovista, o secundarias, cuando los vértices de un eje se encuentren en isovistas diferentes (Fig. 10).

La relación de los puntos en el espacio visual (sensorial) puede ser interpretada en términos sociales del mismo modo que la accesibilidad. Sin embargo, esta inferencia siempre ha de contextualizarse en dinámicas históricas en las que estas relaciones sean significativas. Tradicionalmente, esta lectura se expresa en términos de "privacidad», pero

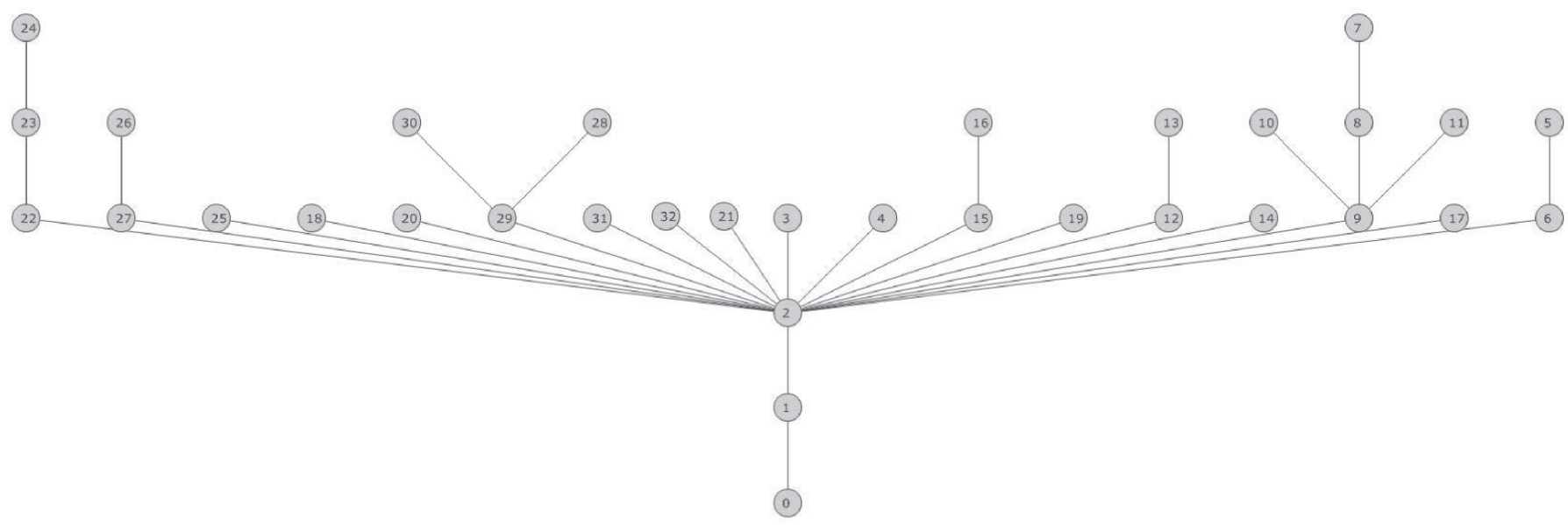

Fig. 8. Gráfico justificado de accesibilidad de la Domus de la Llanuca 


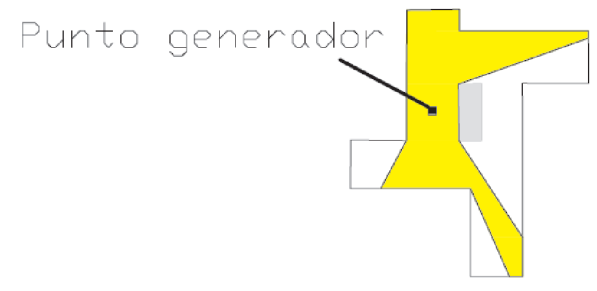

Fig. 9. Representación esquemática de la generación poligonal de una isovista

hemos de tener en cuenta que éste es un concepto social, histórica y culturalmente construido, y por lo tanto no es válido como indicador universal ${ }^{41}$.

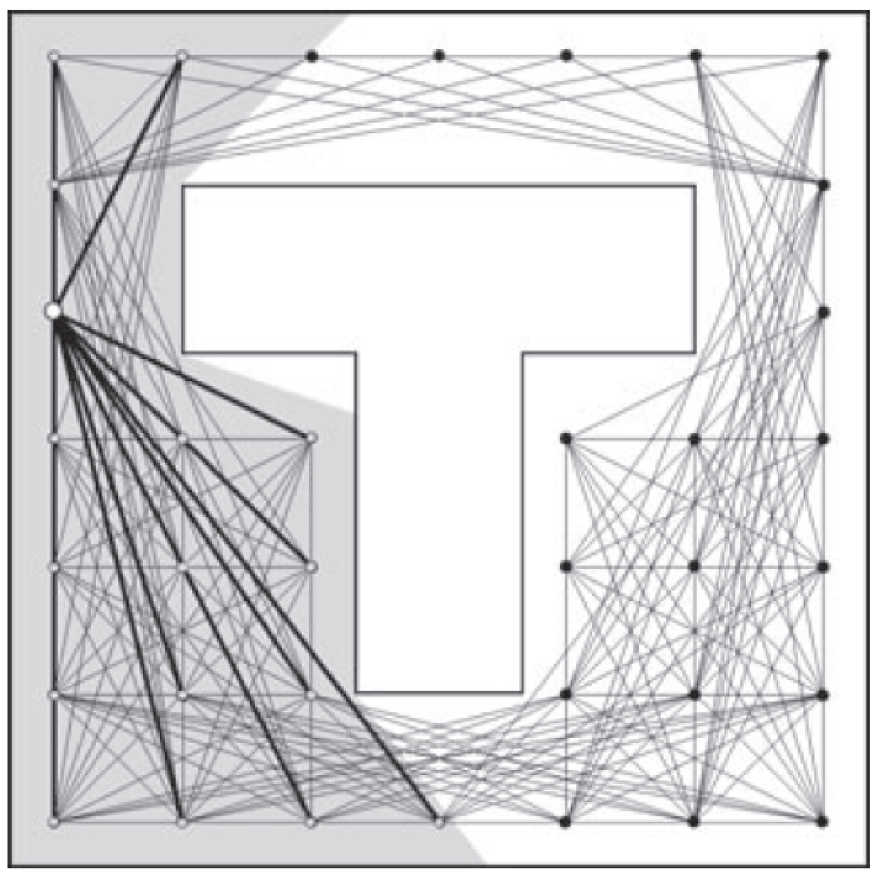

Fig. 10. Representación simple de un gráfico de visibilidad en el que las líneas más gruesas representan ejes de relación primaria.

Además de estas reservas teóricas, también queremos resaltar las dificultades metodológicas que implica la aplicación de gráficos de visibilidad en complejos arqueológicos. En dichos contextos, especialmente en los de época protohistórica o antigua, es casi imposible obtener información espacial acerca de muchos aspectos que podrían alterar las relaciones de visibilidad, como la iluminación, la coloración y por encima de todo, la configuración espacial

${ }^{41}$ Entendemos la potencialidad analítica de este tipo de enfoques al hilo de la aplicación de marcos de pensamiento fenomenológico. A este respecto hemos de aclarar que nuestra crítica no se dirige contra la elaboración teórica general del pensamiento fenomenológico, sino a la aplicación superficial que algunos arqueólogos han propuesto a partir de algunos de sus enunciados. de elementos muebles. La ausencia de estos datos hace que valoremos con escepticismo una aplicación de estas metodologías sobre complejos arquitectónicos conocidos a través de excavaciones arqueológicas, cuya configuración espacial se conozca fundamentalmente a través de reconstrucciones planimétricas.

\section{2. Índices de valor numérico}

Una vez hayamos definido las unidades espaciales que componen un entorno construido, también podemos utilizar una serie de índices analíticos como forma de mesurar o cuantificar sus relaciones sintácticas. Estos índices de relación han sido desarrollados tanto por los ya citados Hillier y Hanson, como por los posteriores trabajos de R. E. Blanton ${ }^{42}$, quien, centrándose en el análisis de espacios domésticos, ha desarrollado una metodología de análisis específico destinada a plantear estrategias espaciales de reproducción social dentro de estructuras domésticas. Este estudio recoge una tradición que se había iniciado en la arqueología antropológica americana de los años setenta con un interesante trabajo de R.L. Hunter-Anderson ${ }^{43}$ sobre la relación formal-social de la estructuras de hábitat protohistóricas.

Dentro de los índices sintácticos propuestos en la obra de Hillier y Hanson encontramos la noción de análisis de tipo local. Este ha sido definido posteriormente como aquel que sirve para mesurar las relaciones que operan entre una unidad espacial y el resto de las unidades de su mismo entorno construido ${ }^{44}$. El índice fundamental para medir este tipo de relaciones es el denominado «valor de control» (CV) ${ }^{45}$. Aplicando los coeficientes detallados por ambos autores ${ }^{46}$, se puede asignar un valor numérico a cada una de ellas (Fig. 11). Aquellas que tengan un valor más alto (UEsp 2) son "controladoras» y aquellas que tengan un valor más bajo son las «controladas» (UEsp que tengan valores en torno a 1 ).

Otro tipo de relaciones sintáctico-espaciales son las llamadas globales, que pueden ser definidas como aquellas que dependen del grado de accesibilidad de las UEsp

\footnotetext{
42 Blanton, R. E. (1994): Houses and households: a comparative study. Plenium Press. En adelante H\&H.

${ }^{43}$ Hunter-Anderson, R. L. (1977): «A theoretical approach to the study of house form». En Binford, L. (Ed.): For theory building in archaeology. New York: 287-315.

${ }^{44}$ Grahame, M. (1999): «Redding the roman house: the social interpretation of saptial order». En Proceedings of TRAC III. Glasgow: 48-73.

${ }^{45}$ SLS, op. cit. supra n. 12: 143-150.

${ }^{46}$ Hemos escogido el ejemplo de la denominada Domus № 2 de la Llanuca (Juliobriga, Cantabria) como ejemplo de la aplicación de los índices detallados en el presente trabajo.
} 
con respecto al punto inicial de su configuración espacial, es decir, el espacio exterior que lo contiene y que sirve de base para el gráfico justificado de accesibilidad. Estas relaciones son mesurables por medio de otro de los índices numérico a los que hacemos referencia, la «asimetría relativa ${ }^{47}$ (AR). Esta se basa en la relación entre la accesibilidad de la unidad espacial y el principio matemático de la simetría-asimetría. Según dicho principio, un espacio será más simétrico si tiene un gran número de relaciones similares. Esto implica que un determinado número de límites habrán de ser cruzados para acceder a él.

Para calcular la asimetría relativa de un espacio, es necesario calcular previamente su profundidad especifica (MDn), que se obtiene tras la aplicación de una sencilla fórmula cuyas variables son fácilmente halladas a través de los datos extraíbles de los gráficos de accesibilidad.

\begin{tabular}{|c|c|c|c|c|c|}
\hline UEsp & $T D n$ & MDn & $A R$ & Int. & $C V$ \\
\hline 0 & 107 & 3,34 & 0,15 & 6,61 & 0,5 \\
\hline 1 & 76 & 2,37 & 0,08 & 11,27 & 1,05 \\
\hline 2 & 47 & 1,46 & 0,03 & 33,06 & 14,58 \\
\hline 3 & 78 & 2,43 & 0,09 & 10,78 & 0,05 \\
\hline 4 & 78 & 2,43 & 0,09 & 10,78 & 0,05 \\
\hline 5 & 107 & 3,34 & 0,15 & 6,61 & 0,5 \\
\hline 6 & 76 & 2,37 & 0,08 & 11,27 & 1,05 \\
\hline 7 & 130 & 4,06 & 0,19 & 5,06 & 0,5 \\
\hline 8 & 99 & 3,09 & 0,13 & 7,4 & 1,25 \\
\hline 9 & 70 & 2,18 & 0,07 & 13,05 & 2,55 \\
\hline 10 & 101 & 3,15 & 0,13 & 7,18 & 0,25 \\
\hline 11 & 101 & 3,15 & 0,13 & 7,18 & 0,25 \\
\hline 12 & 76 & 2,37 & 0,08 & 11,27 & 1,05 \\
\hline 13 & 107 & 3,34 & 0,15 & 6,61 & 0,5 \\
\hline 14 & 78 & 2,43 & 0,09 & 10,78 & 0,05 \\
\hline 15 & 76 & 2,37 & 0,08 & 11,27 & 1,05 \\
\hline 16 & 107 & 3,34 & 0,15 & 6,61 & 0,5 \\
\hline 17 & 78 & 2,43 & 0,09 & 10,78 & 0,05 \\
\hline 18 & 78 & 2,43 & 0,09 & 10,78 & 0,05 \\
\hline 19 & 78 & 2,43 & 0,09 & 10,78 & 0,05 \\
\hline 20 & 78 & 2,43 & 0,09 & 10,78 & 0,05 \\
\hline 21 & 78 & 2,43 & 0,09 & 10,78 & 0,05 \\
\hline 22 & 74 & 2,31 & 0,08 & 11,8 & 0,55 \\
\hline 23 & 103 & 3,21 & 0,14 & 6,98 & 1,5 \\
\hline 24 & 134 & 4,18 & 0,2 & 4,86 & 0,5 \\
\hline 25 & 78 & 2,43 & 0,09 & 10,78 & 0,05 \\
\hline 26 & 107 & 3,34 & 0,15 & 6,61 & 0,5 \\
\hline 27 & 76 & 2,37 & 0,08 & 11,27 & 1,05 \\
\hline 28 & 105 & 3,28 & 0,14 & 6,79 & 0,33 \\
\hline 29 & 74 & 2,31 & 0,08 & 11,8 & 2,05 \\
\hline 30 & 105 & 3,28 & 0,14 & 6,79 & 0,33 \\
\hline 31 & 78 & 2,43 & 0,09 & 10,78 & 0,05 \\
\hline 32 & 78 & 2,43 & 0,09 & 10,78 & 0,05 \\
\hline
\end{tabular}

Fig. 11. Tabla con los valores numéricos de los análisis aplicados a la Domus de la Llanuca

Una ayuda para la generación, tanto de mapas de accesibilidad, como de tablas de valores numéricos a partir de las fórmulas expuestas en los trabajos de Hillier y

${ }^{47}$ Para una explicación específica de este índice analítico vid. Hillier, B; Hanson, J. y Peponis, J. (1984): «What do we mean by building function?». En Powell, J. (Ed.): Designing for building utilization. Londres: 61-67.
Hanson, se encuentra en una aplicación informática desarrollada por B. Manum, un arquitecto noruego, que con la ayuda de dos programadores informáticos ha desarrollado $\mathrm{AGRAPH}^{48}$. Este software ${ }^{49}$, de fácil manejo, es accesible de forma gratuita para cualquier investigador a través de Internet.

Más allá de estos índices que acabamos de explicar, otros autores han desarrollado otras herramientas analíticas. Una de las propuestas más interesantes en este sentido se recoge en el libro de R. E. Blanton, Houses and Households: a comparative study, donde propone dos vertientes en su propuesta de análisis etnoarqueológico de las estructuras domésticas ${ }^{50}$.

La primera es la vertiente "canónica»" ${ }^{51}$ es decir, aquella que analiza la relación del núcleo doméstico con la percepción cosmogónica del ambiente cultural en que se encuentra inscrita. La otra de este tipo de análisis es denominada como «idexical $^{52}$ » y sirve para mesurar aquelos aspectos que sirven como canal comunicativo de mensajes con un significado no-verbal, recibidos por aquellos que habitan la casa.

Con el objetivo de indagar acerca de algunos aspectos de estas vertientes, Blanton propone una serie de índices ${ }^{53}$, cuya base son las formas de representación de los gráficos de accesibilidad. El primero de ellos es el índice de «escala», que es igual al número de unidades espaciales que forman un edificio. Este índice se debe cotejar con el área superficial de la casa, con el fin de establecer o aproximar una densidad de ocupación aproximada ${ }^{54}$.

El índice de «integración» sirve para expresar el tipo de circulación que puede darse en un entorno construido. Se obtiene dividiendo el número de conexiones entre el número de unidades espaciales que componen dicho edificio. Cuanto menor sea el resultado, mayor es la integración con la que es concebido. Los valores altos ${ }^{55}$ nos indican la posibilidad de diversas variantes para acceder a

\footnotetext{
${ }^{48}$ Manum, B.; Rusten, E. y Benze, P. (2005): «AGRAPH. Software for Drawing and Calculating Space Syntex Graphs». En Van Nees, A: (Ed.): Proceedings of the $5^{\text {th }}$ Space Syntax Symposium, June 2005. Techne Press. Amsterdam: 96-103.

${ }^{49}$ Este software ha sido probado por el autor sobre cálculos aplicados a tres casos específicos, en los que los resultados del programa fueron contrastados con los cálculos propios del autor obteniendo una coincidencia plena.

${ }^{50} \mathrm{H} \& \mathrm{H}$, op. cit. supra n. 42: 3-67.

${ }^{51} \mathrm{H} \& \mathrm{H}$, op. cit. supra n. 42: 8.

${ }^{52} \mathrm{H} \& \mathrm{H}$, op. cit. supra n. 42: 9 ss.

${ }^{53} \mathrm{H} \& \mathrm{H}$, op. cit. supra n. 42: 24 ss.

54 Nosotros proponemos también una comparación con los volúmenes de material asociados a dicho entorno doméstico, para contextualizar de forma más completa una aproximación a la densidad de ocupación de la casa.

${ }^{55}$ El valor mínimo es el de 1, porque como hemos dicho una de las características fundamentales de la unidad espacial es que tiene una conexión con otra unidad o con el espacio interior.
} 


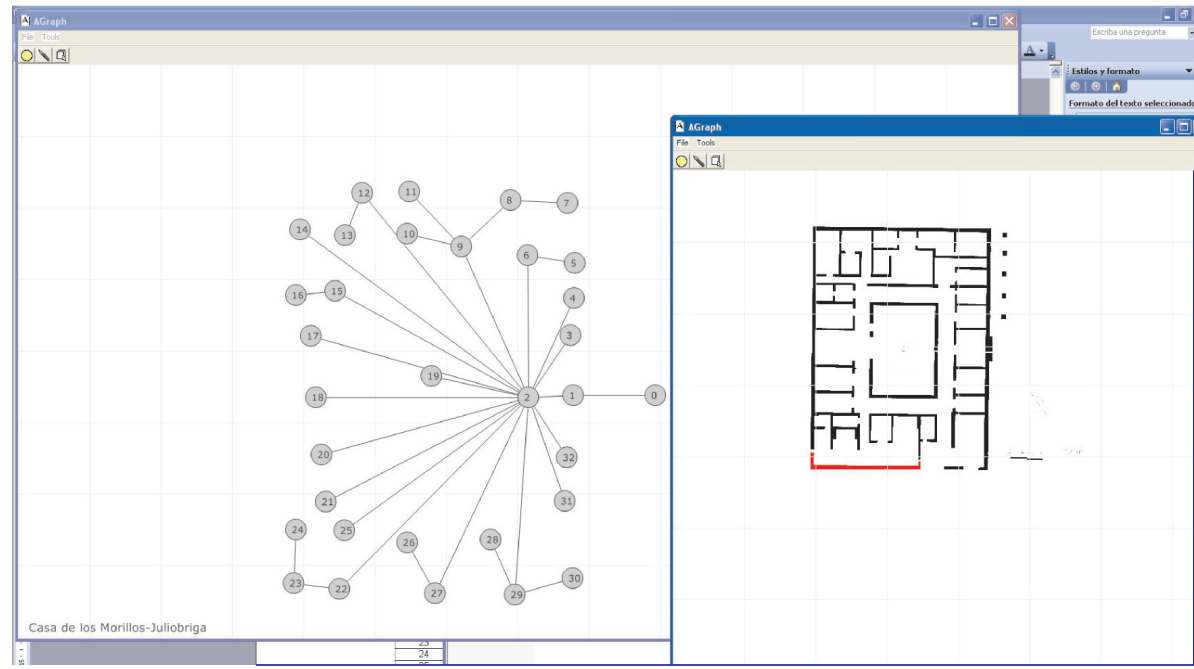

Fig. 12. AGRAPH, aplicación software para el trabajo con análisis tipo space syntax las unidades espaciales. Cuanto menor sea la integración espacial de un edificio, más posibilidades de complejidad y jerarquía interna de la circulación.

El último de los índices es el de «complejidad», utilizado por Blanton para medir la posible variabilidad funcional de los espacios y su presencia dentro del complejo constructivo. Este índice se puede aplicar de forma conjunta (A) o para obtener un valor específico para cada unidad espacial (B). El índice de complejidad A se calcula sumando el número de nodos y conexiones. Por el contrario, el índice de complejidad $\mathrm{B}$ señala el número de espacios, que deben cruzarse para acceder a una unidad espacial desde el exterior.

La aplicación de este tipo de análisis sobre el ejemplo que hemos seleccionado, la domus No 2 de la Llanuca (Juliobriga, Cantabria), excavada por A. García y Bellido, y estudiada de forma exhaustiva junto con otras casas del municipium $^{56}$, nos permite vislumbrar algunas características de sus configuración espacial y conocer algunos aspectos de su diseño arquitectónico que trascienden lo meramente descriptivo o tipológico.

Del cotejo de estos datos podemos extraer una serie de conclusiones de tipo sintáctico-espacial. La UEsp 2 tiene un valor de control (CV) (Fig. 11) muy superior al resto de las habitaciones, se trata de un espacio dominante dentro de la configuración espacial. Además, un repaso al resto de los valores nos indica que no existe una jerarquización interna muy grande con respecto al control ejercido por otras UEsp. Su gráfico de accesibilidad nos muestra una configuración altamente simétrica. El valor del índice de

${ }^{56}$ Iglesias Gil, J. (Ed. 2000): Arqueología en Juliobriga. Universidad de Cantabria. Santander. Con Bibliografía. integración es relativamente bajo, lo que nos indica un patrón de circulación bastante rígido.

El índice de escala y el índice de complejidad A, en relación al área de extensión de la $\mathrm{casa}^{57}$, puede ser interpretado como reflejo de una cierta sencillez funcional, muy en sintonía con las construcciones domésticas de contextos culturales pre-industriales, en los que la especialización funcional de las UEsp, suele ser reducida ${ }^{58}$. Aunque también debemos indicar que, dentro del contexto de otros entornos construidos pre-industriales, nos encontramos ante un prototipo de especialización funcional eleva$\mathrm{da}$, que se puede equiparar a la de otras construcciones propias de contextos de elite, como la casa colonial hispano-americana ${ }^{59}$.

Traducido en términos sociales, esto podría indicarnos una estructuración del hábitat relativamente simple, basa$\mathrm{da}$ en un término superior que controla de forma directa a una comunidad doméstica, sin que existan grandes diferencias internas entre el resto de miembros, por lo menos en términos espaciales. Asimismo, los índices de escala y complejidad, están indicando un patrón de actividad en el que todos los miembros de la unidad doméstica comparten espacios de habitación de forma mayoritaria.

Lecturas sintácticas como estas pueden ser muy útiles para completar estudios sobre determinados aspectos de la historia social y cultural, que en el caso concreto de las comunidades de época romana, han sido abordados tradicionalmente sobre la base de la documentación textual y de

\footnotetext{
${ }^{57}$ En el caso de la Domus de la Llanuca, en torno a los $220 \mathrm{~m} 2$.

${ }^{58}$ Frente a entornos construidos de época post-industrial, donde la especialización de los espacios suele ser la nota predominante.

${ }^{59}$ Vid. Funari, P. P. y Zarankin, A. (2003), op cit. supra n. 17: Fig. 4.
} 


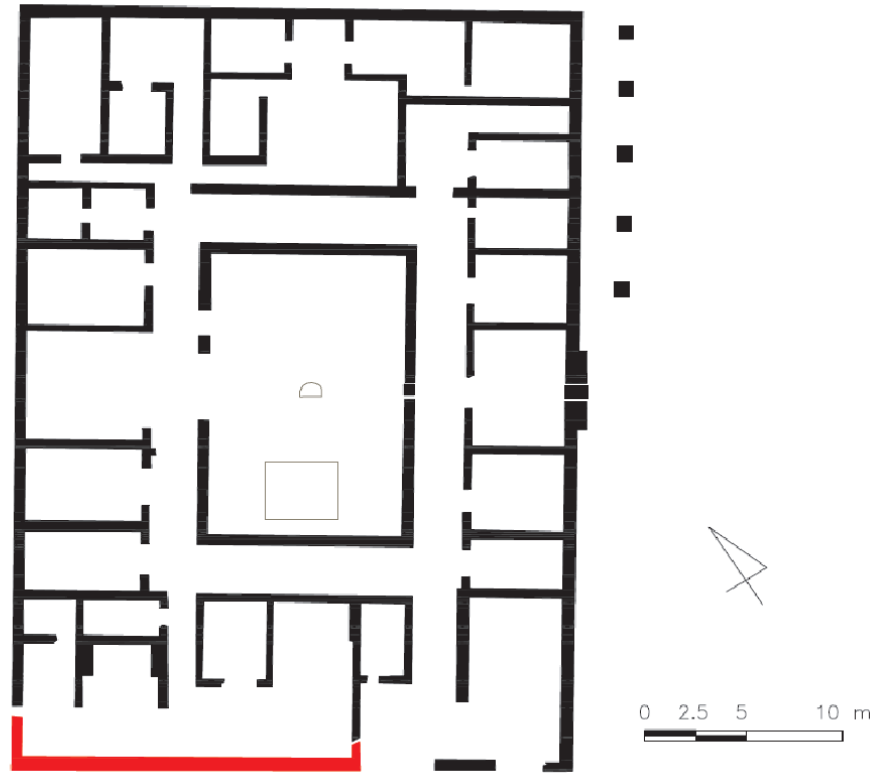

\begin{tabular}{|c|}
\hline ÍNDICE DE ESCALA: 32 \\
\hline ÍNDICE DE INTEGRACIÓN: 1,031 \\
\hline ÍNDICE DE COMPLEJIDAD A: 65 \\
\hline ÍNDICE DE COMPLEJIDAD B: 3,3 \\
\hline
\end{tabular}

Fig. 13. Algunos de los valores sintácticos de la Domus de la Llanuca-Juliobriga. El índice de complejidad B es una media de los diferentes valores asignados a las distintas UEsp

paralelos planimétricos. Pese a ello, antes de proponer una contextualización profunda de estos datos (algo que trasciende del objeto de este trabajo), creemos conveniente la exposición de una serie de cuestiones metodológicas que en nuestra opinión plantean matices importantes a las conclusiones previas que acabamos de exponer.

\section{CUESTIONES METODOLÓGICAS I: SPACE SYNTAX VS ARCHAEOLOGIA DELL'ARCHITECTURA}

La aplicación de éstos índices analíticos para el estudio de entornos construidos en contextos arqueológicos ha suscitado dos tipos de reacciones historiográficas más o menos diferenciables. En primer lugar, aquellos que desde posiciones teóricas cercanas al posprocesualismo arqueológico o la teoría crítica marxista (agrupados recientemente por $\mathrm{V}$. Fernánde $z^{60}$ bajo la denominación de "arqueologías críticas») las han aceptado abiertamente ${ }^{61}$, convirtiéndolas en

${ }^{60}$ Fernández, V. (2006): Una arqueología crítica: ciencia, ética y política en la construcción del pasado. Crítica. Barcelona.

${ }^{61}$ Frente a la denominada arqueología espacial procesual, que en su orientación hermenéutica, diverge de la utilización de la sintaxis espacial por parte de los arqueólogos «críticos». muchos casos en protagonistas principales de estudios monográficos ${ }^{62}$.

La otra reacción historiográfica mayoritaria ha sido mantenida por diversos sectores vinculados con el procesualismo epistemológico, centrado en el registro empírico de datos que han visto con recelo, y en algunos casos con abierto rechazo ${ }^{63}$, las interpretaciones sociales derivadas de este tipo de metodología.

A medio camino entre la aceptación acrítica de los mismos y el rechazo absoluto de sus posibilidades interpretativas, pretendemos exponer los principales problemas suscitados con motivo de la reflexión metodológica derivada de nuestra propia investigación ${ }^{64}$. De este modo queremos hacer partícipe al lector de nuestros planteamientos acerca de las limitaciones de la sintaxis espacial como herramienta de análisis histórico, con el objetivo de establecer un marco teórico-metodológico desde el que poder ofrecer nuevas aplicaciones destinadas a enriquecer la interpretación arqueológica de entornos arquitectónicos ${ }^{65}$.

Un primer punto problemático ${ }^{66}$ se nos plantea al considerar los métodos de la sintaxis espacial en comparación con el análisis estratigráfico de los elementos constructivos de edificios históricos o protohistóricos. Este tipo de análisis arqueológico resulta especialmente útil para entender de forma detallada el proceso de construcción, remoción y (en algunos casos) abandono de edificios o de determinadas partes.

Es por lo tanto otra forma de aproximarse a la biografía social ${ }^{67}$ de una construcción a lo largo de un periodo histórico. Pese a que, por si sola, no sirve para explicar las causas históricas de ese desarrollo ${ }^{68}$, nos ofrece un panorama de los entornos construidos como elementos dinámicos. Esta forma de concebir la arquitectura $-\mathrm{y}$ los

\footnotetext{
${ }^{62}$ Grahame, M. (2000), op. cit. supra n. 15. Zarankin, A. y Niro, C. (2007), op. cit. supra n. 17.

${ }^{63}$ Una crítica de este tipo la tenemos en Allison, P. (1999): «Introduction». En Allison, P. (Ed.): The Archaeology of Household Activities. Routledge. Londres: 410. Cutting, M. (2003): "The use of spatial analysis to study prehistoric settlement architecture», Oxford Journal of Archaeology. 22 (1): 1-21.

${ }^{64}$ Centrada en el análisis de los espacios domésticos de época romana en varios puntos de la Península y el Mediterráneo.

${ }^{65}$ Brogiolo, G.P. (2007): «Dall'Archaeologia dell'architecttura all'Archaeologia della complessitá». Pyrenae. 38 (1): 2-39.

${ }^{66}$ Ya intuido en un reciente trabajo de A. Azkarate (2008): «La arqueología de la arquitectura en el siglo XXI». Arqueología de la Arquitectura. 5: 11-13.

${ }^{67}$ Kopytoff, I. (1986): «The cultural biography of things: commoditization as process". En Appaidurai, A. (Ed.): The social life of things. Commodities in cultural perspective. University Press. Cambridge: 64-91.

${ }^{68}$ Nos referimos a las "limitaciones históricas» a las que hace referencia L. Caballero (1996): «El análisis estratigráfico de construcciones históricas». En Escribano, C. y Caballero, L. (Coor.): Arqueología de la arquitectura: el método arqueológico aplicado al proceso de estudio y de intervención en edificios históricos. Junta de Castilla y León. Valladolid: 55-74.
} 
elementos sociales y culturales a ella asociados- que nos ofrece el análisis estratigráfico de los edificios contrasta con el análisis sintáctico de los espacios, caracterizado por aplicarse a momentos estáticos de la secuencia temporal ${ }^{69}$.

Las raíces estructuralistas sobre las que, según hemos argumentado, se asienta el modelo teórico de la sintaxis espacial produce inferencia de tipo anacrónico, siendo incapaz no ya de explicar, sino siquiera de reflejar interpretaciones desde una perspectiva dinámica, de cambio histórico-social. En otras palabras, ofrece datos interesantes para la interpretación de un segmento temporal determinado pero es incapaz de reflejar cambios en una secuencia diacrónica como la ofrecida por la aplicación de la estratigrafía como método analítico, del mismo modo que le ocurre al estructuralismo antropológico ${ }^{70}$.

Este carácter inherente al tipo de análisis planteado por la sintaxis espacial explica, en parte, su rápida extensión y aceptación por otras disciplinas que, en principio, no están obligadas a incluir una explicación diacrónica del cambio social y cultural, aspecto que en nuestra opinión es indisoluble del pensamiento histórico y por extensión del arqueológico, especializándolo frente a otras ciencias sociales.

Frente a este problema, algunos recientes trabajos han mostrado una voluntad de combinar ambas metodologías para conseguir un beneficio mutuo, dotando a la sintaxis espacial de una cierta capacidad de expresión diacrónica, a través del ejercicio sobre varios momentos o fases deducibles de la secuencia estratigráfica de los edificios ${ }^{71}$. Se trata éste de un camino ineludible para la arqueología de la arquitectura en el futuro y a buen seguro que habrá de producir interesantes resultados. Las posibilidades de enriquecimiento mutuo, como vía para reducir las «limitaciones históricas» de la arqueología de la arquitectura, así como forma de dotar de dinamismo cronológico a los análisis de sintaxis espacial, han quedado probadas, por citar tan sólo un ejemplo, en un reciente trabajo de S. Gutiérrez y P. Cánovas $^{72}$, donde se argumenta una novedosa propuesta interpretativa para el conjunto monumental que incluye la denominada basílica, sobre la base del análisis de la sintaxis espacial de una de sus fases constructivas.

\footnotetext{
${ }^{69}$ Normalmente aplicados a un determinado trazado planimétrico, o bien sobre una sucesión de trazados sin conexión diacrónica inmediata.

${ }^{70}$ Hodder, I. y Hudson, S. (2003): Reading the Past: Current Approaches to Interpretation in Archaeology. University Press. Cambridge: 52-75.

${ }^{71}$ Ayán Vila, X. M.; Blanco Rotea, R. y Mañana Borrazás, P. (2003), op. cit. supra n. 10: 2-8.

${ }^{72}$ Gutiérrez, S. y Cánovas, P. (2009): «Construyendo el siglo VII: arquitecturas y sistemas constructivos en el Tolmo de Minateda». En Caballero, L.; Mateos, P. y Utrero, M. ${ }^{a}$ Á. (Eds.): El siglo VII frente al siglo VII: Arquitectura. Anejos de AEspA. LI. CSIC. Madrid: 91-132.
}

Sin embargo no es menos cierto, al menos desde nuestra experiencia, que esta complementación de metodologías topa con una serie de problemas, especialmente patentes cuando abordamos el análisis de edificios antiguos o prehistóricos. Estos problemas se resumen fundamentalmente en dos: la ausencia de alzados que nos sirvan para inferir secuencias constructivas detalladas y la falta de secuencias estratigráficas en edificios excavados. A este respecto, el principal obstáculo con el que nos topamos es la distorsión producida por criterios de documentación que, al no tener en cuenta los procesos de formación del registro arqueológico ${ }^{73}$, dificultan la inferencia de nivel de ocupación en esta clase de edificios.

Esta cuestión es especialmente relevante para la arqueología de los espacios residenciales, en la que el término "arquitectura» normalmente sirve para referirse a los trazados planimétricos. El propio carácter de los vestigios materiales dentro de estos contextos arqueológicos fuerza en la mayoría de los casos a trabajar de esta forma. Es por esta razón por lo que la investigación de los aspectos sociales derivados de estos entornos construidos se ha concebido fundamentalmente desde una perspectiva bidimensional.

Ejemplo de esta problemática es también la domus de la Llanuca, excavada en su mayor parte por A. García y Bellido $^{74}$ sin criterios de documentación estratigráfica precisa. De esta forma, nuestra esquemática propuesta de secuencia muraria no puede ser apoyada en una secuencia estratigráfica que permita aportar datos relativos a las fases de ocupación asociadas a las reformas detectadas en su estructura.

La técnica edilicia detectada se puede dividir básicamente en dos tipos en cuanto al tipo de paramento utilizado en la cimentación de la estructura. Un primer tipo (A) estaría compuesto por bloques de arenisca trabajados de forma irregular ${ }^{75} \mathrm{y}$ alargada. Este tipo de zócalos o cimientos — no existe una indicación segura de los niveles topográficos de las zanjas de cimentación- se encuentra trabado por medio de un mortero de escasa calidad. El tipo (B) está compuesto por sillares calizos careados de forma más homogénea y trabados por medio del mismo mortero. También es frecuente encontrar orificios en los cimientos

\footnotetext{
${ }^{73}$ Schiffer, M. B. (1987): Formation processes of archaeological record. New México University Press. Albuquerque.

${ }^{74}$ García y Bellido, A.; Fernández de Avilés, A. y García Guinea, M. A. (1970): Excavaciones y exploraciones arqueológicas en Cantabria. Anejos de AEspA IV. Instituto Español de Arqueología. CSIC. Madrid.

${ }^{75}$ Fernández Vega, P. A. (1993): Arquitectura y urbanistica en la ciudad romana de Juliobriga. Universidad de Cantrabria. Torrelavega: 36.
} 
pétreos de dichas estructuras, interpretados como soportes de vigas de madera, documentadas desde época protohistórica $^{76}$, que debieron haber formado parte del sistema de ensambladura que servía de soporte a los alzados realizados mediante la utilización de la tierra como elemento constructivo.

El empleo de diversas técnicas de construcción con barro, frecuentemente en la arquitectura doméstica meseteña de época romana durante los periodos alto y bajoimperial, también debió ser la técnica edilicia más usada en los alzados de Juliobriga. La escasa atención prestada a la documentación de derrumbes durante el proceso de su excavación nos impide tener ningún tipo de información precisa sobre su técnica y tipología.

La mayor parte de las cimentaciones documentadas en la domus No 2 pertenecen al tipo B, siendo la única casa de Juliobriga en la que se documenta este tipo de paramento $^{77}$. Sin embargo, ante la imposibilidad de asociar un tipo paramental concreto a las escasas remociones reconocibles en su estado actual, como es el cierre de su patio porticado, nos es imposible establecer una secuencia constructiva detallada.

Más allá de estos problemas, y ante la ausencia de una lectura estratigráfica detallada, creemos que esta secuencia no debió ser muy compleja, con una uniformidad constructiva argumentable por medio del detallado estudio metrológico publicado por P. A. Fernández $\mathrm{Vega}^{78}$, quien defendía una unidad de concepción en el trazado planimétrico de la casa, con una estructuración interna que perduró en gran medida inalterada durante la mayor parte de su ocupación.

\section{CUESTIONES METODOLÓGICAS II: SPACE SYNTAX VS ARTEFACT ASSEMBLAGES}

Otro de los problemas metodológicos que plantea la aplicación de análisis tipo space syntax es su absoluta desatención ${ }^{79}$ a aquello que Rapoport ${ }^{80}$ denominaba «elementos no fijos» (non-fixed elements). Dichos elementos o artefactos de tipo mueble también afectaban a la configuración espacial de los entornos construidos y a los sistemas

${ }^{76}$ Vela Cossío, F. (2002): Espacio doméstico y arquitectura del territorio en la prehistoria peninsular: tipología y razón constructiva en la arquitectura celtibérica. Tesis doctoral leída en el Departamento de Prehistoria de la Universidad Complutense. Madrid: 170-182.

${ }^{77}$ Fernández Vega, P. A. (1993), op. cit. supra n. 75: 40

${ }^{78}$ Fernández Vega, P. A. (1993), op. cit. supra nota 75: 42-46.

${ }^{79}$ En este punto coincidimos plenamente con la crítica de P. Allison (1999), op. cit. supra n. 63.

${ }^{80}$ Rapoport, A. (1969): House form and culture. Londres. Id. (1990): The meaning of the built environment: a non-verbal communication approach. niversity of Arizona. Tucson. de actividades asociados a ellos ${ }^{81}$. En esta línea interpretativa, la denominada household archaeology ${ }^{82}$, ha propiciado un desarrollo metodológico destinado al análisis de los diversos elementos muebles contenidos en el registro arqueológico de las unidades domésticas. En ella confluyen dos aspectos muy importantes, la sistematización conjunta de la cultura material doméstica y la conciencia de los diferentes contextos de uso documentados a partir del estudio (etnoarqueológico) de los procesos de formación del registro arqueológico ${ }^{83}$.

Este tipo de enfoques fueron desarrollados por el procesualismo arqueológico, que sobre la base del valor espacial $^{84}$ de los diferentes artefactos arqueológicos en relación a su proveniencia ${ }^{85}$, desarrolló toda una serie de estrategias para el análisis de los mismos en relación a un contexto espacial interior ${ }^{86}$.

La documentación proporcionada por estos aspectos puede ser, en algunos contextos, muy determinante a la hora de interpretar el significado social y las actividades realizadas en una unidad espacial. Lamentablemente, la ausencia de un contexto arqueológico preciso en la domus No 2 de la Llanuca nos impide realizar una aproximación de este tipo que sirva como ejemplo de esta problemática metodológica.

Pese a ello contamos con una serie de casos documentados en ámbitos urbanos de época romana, que nos sirven para ilustrar el modo en que el conocimiento detallado de la cultura material mueble depositada en el registro de una unidad espacial puede servir para matizar modelos establecidos mediante criterios planimétrico-tipológicos.

Un estudio publicado por J. Berry ${ }^{87}$ proponía una reflexión muy sugerente acerca de cómo la falta de integración de los datos provenientes de contextos materiales muebles nos puede hacer caer en la distorsión de interpre-

81 De forma especialmente relevante a las relaciones visuales como hemos destacado en nuestra crítica a la utilización de gráficos de visibilidad en el análisis de yacimientos arqueológicos.

${ }^{82}$ Allison, P. (2008): Voz «Household archaeology». En Pearsall, D. (Coor.): Encyclopedia of Archaeology. Academic Press: 1449-1458.

83 Para una discusión metodológica vid. Binford, L. (1981): «Behavioral archaeology and the "Pompeii Premise». JAR 37 (3): 195-208. Schiffer, M. B. (1985): «Is there a 'Pompeii Premise' in archaeology?». JAR 41 (1): 18-41.

${ }^{84}$ Hodder, I. y Orton, C. (1974): Spatial Archaeology. University Press. Cambridge.

${ }^{85}$ Schiffer, M. B. (1987): Formation processes of archaeological record. University of New México Press. Albuquerque. 3-19.

${ }^{86}$ Kent, S. (Ed.) (1990): Domestic architecture and the use of space. Cambridge University Press. New York. Heitala, H.J. (1984): "Intrasite analysis: a brief review». En Heitala, H. J.(Ed.): Intrasite Spatial Analysis in Archaeology. Cambridge University Press. Londres: 1-4.

${ }^{87}$ Berry, J. (1997): «Household artefacts: re-interpreting roman domestic space». En Laurence, R. y Wallace-Hadrill, A. (Eds.): Domestic space in the roman world: Pompeii and Beyond. Suppl. JRA. 22: 155-195. 
tar social y económicamente espacios completamente diferentes como ámbitos similares. Una serie de ejemplos pompeyanos nos sirven para plantear problemáticas análogas en un contexto equiparable al caso juliobrigense en términos metodológicos.

En ellos la integración de los datos del instrumentum domesticum nos ilustra sobre la complejidad del espacio doméstico romano, en el que la disposición espacial de los muros de las casas, por si solos, no nos sirve para entender la relación entre ocio y trabajo cotidiano, o entre la casa romana como hogar y como lugar de industria-producción ${ }^{88}$.

Si la configuración arquitectónica nos puede ofrecer una visión de la estructuración social reflejada en sus espacios, la documentación mueble nos permite crear una visión más completa del funcionamiento cotidiano de la misma. Como en el caso de la imagen que se reproduce en la figura 14, un conocimiento más detallado de la cultura material, debidamente depositada y secuenciada, nos puede ofrecer una visión mucho más certera de los parámetros sociales y culturales que regían las actividades dentro de los edificios.

El trabajo con este tipo de datos permite romper con la idea de que el espacio social, aquel que tratamos de caracterizar por medio de la sintaxis espacial, en una entidad circunscrita únicamente a la materialidad de la construcción. Esta perspectiva es especialmente importante en el caso de arquitecturas protohistóricas o antiguas que, por no haber dejado constancia material en el registro arqueológico, han sido ignoradas o excluidas de la investigación, pese a tener un amplio registro de materiales muebles que pueden servir para apoyar su existencia.

Estos ejemplos nos sirven para argumentar de qué modo el conocimiento detallado de la cultura material mueble puede introducir elementos muy importantes a la hora de interpretar la estructuración espacial de edificios históricos en clave social. Hemos introducido este apartado a modo de reflexión acerca de la variabilidad inherente al análisis arqueológico de espacios construidos. Teniendo en cuenta esta perspectiva de complejidad social y funcional, la sintaxis espacial puede ser utilizada como una herramienta de análisis especialmente útil para obtener lecturas socio-estructurales (de las diferentes identidades que se dan cita en los ámbitos domésticos) y dinámicas de poder latentes en el seno de los entornos construidos. Sin embargo, esa misma complejidad también obliga a la confrontación de éstos índices con datos proporcionados por otras tradiciones analíticas.

${ }^{88}$ Allison, P. (2004): Pompeii households: Analysis of the material culture. Monograph 42. Cotsen Intitute of Archaeology. UCLA. Los Angeles.

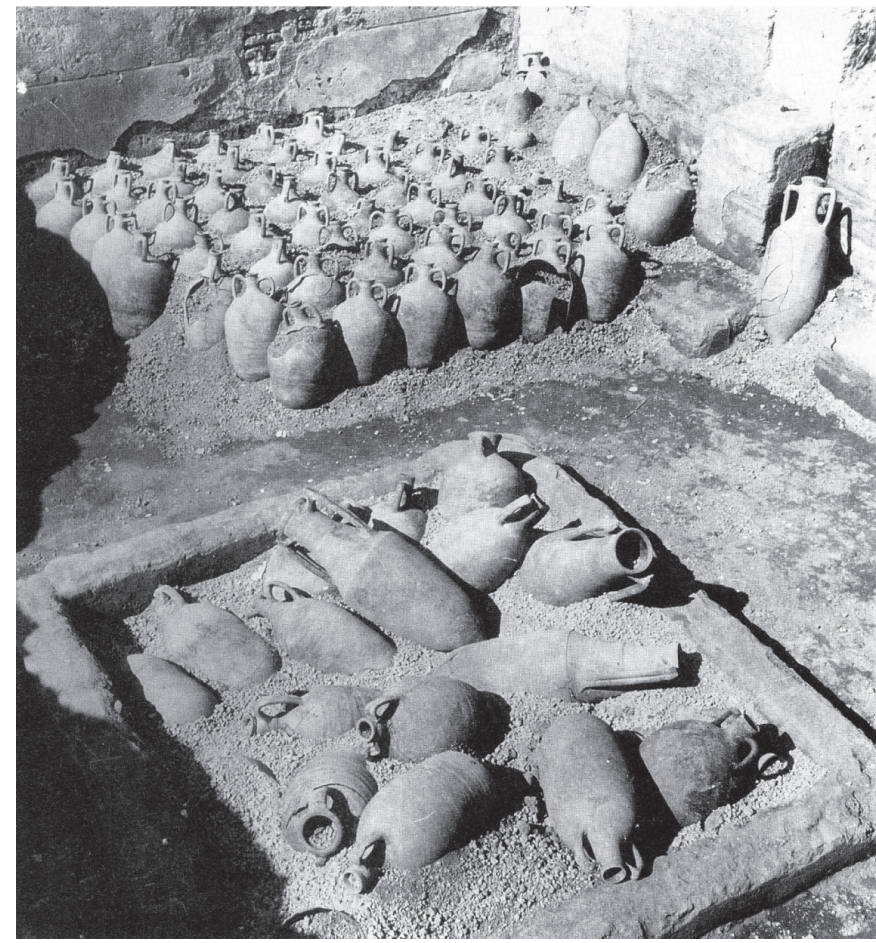

Fig. 14. Atrium de la Domus I. 9.12 (Pompeya) en el momento de su excavación tomada de Berry, J. (1997)

\section{CONSIDERACIONES FINALES: INTEGRANDO DATOS E INTERPRETACIÓN HACIA UNA ARQUEOLOGÍA DE LO COMPLEJO}

La arqueología (de la arquitectura o cualquier otra) se encuentra inmersa en un profundo debate epistemológico, reflejo de las discusiones teóricas generadas por el mundo global de la posmodernidad. Pocos son los conceptos metodológicos que pueden escapar a una crítica más o menos parcial. Más allá de esto resulta plausible pensar que dentro de este panorama variado, las propuestas interpretativas habrán de plantear progresivamente matices más sutiles y complejos. En nuestra opinión esto implica que los datos sobre los que se han de sustentar estas interpretaciones sean más fiables, cualitativa y cuantitativamente hablando, a fin de poder sustentar de forma adecuada esa complejidad epistemológica a la que hacemos referencia.

La sintaxis espacial ofrece un método de producción de datos fiables, aplicables a un gran número de casos de estudio, con relativamente poco esfuerzo. Sin embargo, esto no significa que las interpretaciones inferidas a partir de estos mismos datos sean más sólidas que las extraídas a partir de la aplicación de otras metodologías.

Desde nuestra perspectiva, la utilización de las capacidades analíticas de los software tipo SIG ofrecen una herramienta de trabajo que permite la integración de datos generados a partir de lecturas analíticas de diversa índole, 


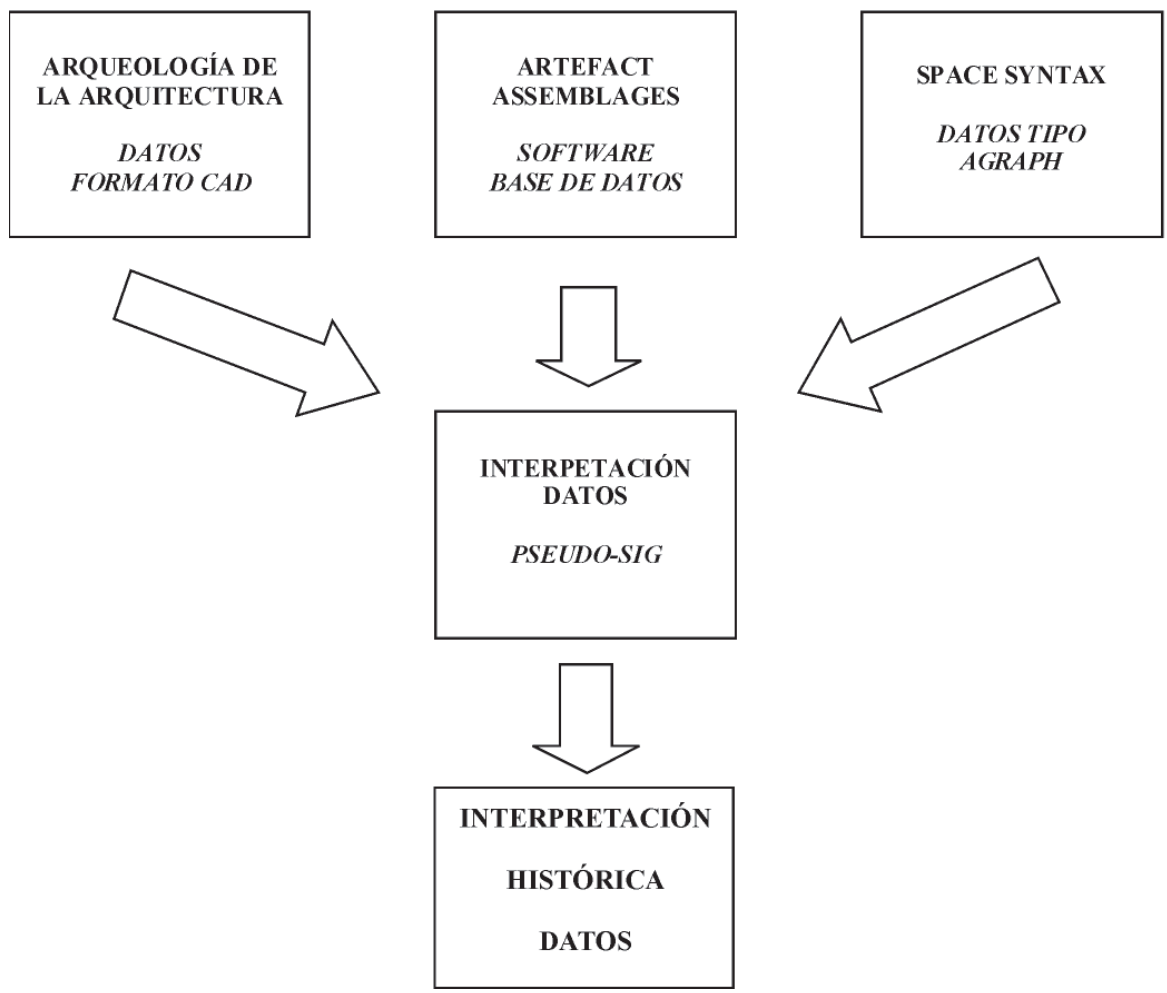

Fig. 15. Cuadro resumen de la propuesta metodológica para la integración de datos arqueológicos

lo que facilita una complementariedad de enfoques metodológicos. A este respecto, hemos de reseñar que la aplicación de este tipo de herramientas informáticas para el análisis interior de edificios ya cuenta con algunos hitos bibliográficos, que de forma incipiente nos hacen aventurar grandes perspectivas de innovación científica ${ }^{89}$.

El análisis constructivo de la arquitectura ha contado con los programas tipo CAD como una herramienta de trabajo fundamental, mientras que los análisis de contextos materiales y las secuencias estratigráficas tienen en las bases de datos el software fundamental para su gestión. Las nuevas aplicaciones informáticas generadas a partir de la aplicación de análisis tipo space syntax constituyen el otro vértice fundamental de nuestra propuesta metodológica para el futuro.

La utilización de herramientas SIG ofrece además la posibilidad de relacionar estos datos con otros procedentes de la vinculación de los entornos construidos estudiados con otros provenientes del análisis del paisaje desde una perspectiva arqueológica, enmarcándolos en una dinámica histórico-geográfica concreta.

89 Para una revisión de algunas recientes aportaciones se puede consultar la revista Internet Archaeology, que en su número 24 ofrece un monográfico sobre la aplicación de herramientas SIG al análisis material de entornos arquitectónicos. Allison, P. (Coor. 2008): «Dealing with legacy data». Internet Archaeology 24. http://intarch.ac.uk/journal/issue24/
Pese a la amplia batería de posibilidades analíticas que acabamos de esbozar, lo cierto es que la mayor parte de las aplicaciones arqueológicas de este tipo de métodos han sido protagonizadas por investigadores que se encuentran en los primeros pasos de su carrera profesional ${ }^{90}$. Son (somos) pocos todavía los que defienden las posibilidades interpretativas derivadas de la utilización de índices de sintaxis espacial en el análisis arqueológico, por lo que habrá que esperar a la aplicación de un programa continuado y coherente de investigación para poder evaluar de forma ponderada la validez científica de este tipo de métodos, sobre todo teniendo en cuenta el ambicioso proyecto intelectual que plantea.

\section{Bibliografía}

Allison, P. (1999): «Introduction». En Allison, P. (Ed.): The Archaeology of Household Activities. Routledge. Londres: 4-10.

Allison, P. (2004): Pompeii households: Analysis of the material culture. Monograph 42. Cotsen Intitute of Archaeology. UCLA. Los Angeles.

Allison, P. (2008): Voz «Household archaeology». En Pearsall, D. (Coor.): Encyclopedia of Archaeology. Academic Press: 1449-1458.

Allison, P. (Coor. 2008): «Dealing with legacy data». Internet Archaeology 24. http://intarch.ac.uk/journal/issue24/

Ayán Vila, X. M. (2003): «Arquitectura como tecnología de construcción social». Arqueología de la Arquitectura. 2: 17-24.

${ }^{90}$ Casi todos ellos de origen anglosajón o vinculados de alguna forma a sus ambientes académicos, sobre todo a nivel teórico. 
Ayán Vila, X. M. ; Blanco Rotea, R. y Mañana Borrazás, P. (2003): «Archaeotecture: seeking a new archaeological vision of architecture». En Ayán Vila, X. et alii (Eds.): Archaeotecture: Archaeology of Architecture. BAR International Series 1175. Archaeopress. Oxford: 1-15.

Benedikt, M. L. (1979): «To take hold of space: isovists and isovist fields». Environment and Planning B. 6: 47-65.

Benedikt, M. L. y Burnham, C. A. (1985):«Perceiving architectural space: from optic rays to isovists». En Warren, W. H. y Shaw, R. E. (Eds.): Persistence and Change. Londres: 34-48.

Berry, J. (1997): «Household artefacts: re-interpreting roman domestic space». En Laurence, R. y Wallace-Hadrill, A. (Eds.): Domestic space in the roman world: Pompeii and Beyond. Suppl. JRA. 22: 155-195.

Binford, L. (1981): «Behavioral archaeology and the «Pompeii Premise». JAR 37 (3): 195-208.

Blanton, R. E. (1994): Houses and households: a comparative study. Plenium Press.

Bourdieu, P. (1973): «The Berber house». En Douglas, M. (Ed.): Rules and meaning. Penguin. Suffolk: 98-110.

Brogiolo, G.P. (2007): «Dall'Archaeologia dell'architecttura all'Archaeologia della complessitám. Pyrenae. 38 (1): 2-39.

Caballero Zoreda, L. y Escribano Velasco, C. (1996): «El método arqueológico aplicado al proceso de estudio e intervención en edificios históricos». En Arqueología de la Arquitectura. Junta de Castilla y León. Burgos.

Calafell, E. (2000): Las unités d'habitation de Le Corbusier: aspectos formales y constructivos. Fundación Caja de Arquitectos. Barcelona.

Cutting, M. (2003): «The use of spatial análisis to study prehistoric settlement architecture» Oxford Journal of Archaelogy. 22 (1): 1-21.

Fernández Martínez, V. (2006): Una arqueología crítica: ciencia, ética y politica en la construcción del pasado. Crítica. Barcelona.

Fernández Vega, P. A. (1993): Arquitectura y urbanistica en la ciudad romana de Juliobriga. Universidad de Cantrabria. Torrelavega.

Foucault, M. (1980): «El ojo del poder». En Bentham, J: El Panóptico. Ed. La Piqueta. Madrid.

Fumadó, I. (2007): «Introducción al estudio de los baños domésticos de tradición fenicio-púnica». Saguntum. 39: 103-115.

Funari, P. P. y Zarankin, A. (2003): «Social archaeology of housing from a Latin American perspective: A case study». Journal of Social Archaeology. 3: 23-45.

García y Bellido, A.; Fernández de Avilés, A. y García Guinea, M. A. (1970): Excavaciones y exploraciones arqueológicas en Cantabria. Anejos de AEspA IV. Instituto Español de Arqueología. CSIC. Madrid.

Gaston, C. (2005): Mies: el proyecto como revelación del lugar. Barcelona.

Grahame, M. (1999): «Reading the roman house: the social interpretation of spatial order». Proceedings of III TRAC Conference. Cruitnhe Press. Glasgow: 48-74.

Grahame, M. (2000): Reading Space: Social Interaction and Identity in the Houses of Roman Pompeii. BAR Int. Ser. 886. Archaeopress. Oxford.

Gutiérrez, S. y Cánovas, P. (2009): «Construyendo el siglo VII: arquitecturas y sistemas constructivos en el Tolmo de Minateda». En Caballero, L.; Mateos, P. y Utrero, M. A. (Eds.): El siglo VII frente al siglo VII: Arquitectura. Anejos de AEspA. LI. CSIC. Madrid: 91-132.

Heitala, H.J. (1984): «Intrasite analysis: a brief review». En Heitala, H.J.(Ed.): Intrasite Spatial Analysis in Archaeology. Cambridge University Press. Londres: $1-4$.

Henaff, M. (1991): Claude Lévi-Strauss et l'anthropologie structurale. Belfond. Paris.

Hillier, B. (1996): Space is the machine. University Press. Cambridge.

Hillier, B. (1999): «Centrality as a process: accounting for attraction inequalities in deformed grids». Urban design. 4 (3-4): 107-127.

Hillier, B. y Hanson. J. (1984): The Social Logic of Space. University Press. Cambridge.

Hillier, B. Hanson, J. y Graham, H. (1987): «Ideas are in things: an application of space syntax method to discovering house genotypes». Environment and Planning B: Planning and Design. 14: 363-385.

Hillier, B; Hanson, J. y Peponis; J. (1984): «What do we mean by building function?». En Powell, J. (Ed.): Designing for building utilization. Londres: 6167.

Hodder, I. y Hudson, S. (2003): Reading the Past: Current Approaches to Interpretation in Archaeology. University Press. Cambridge.
Hodder, I. y Orton, C. (1974): Spatial Archaeology. University Press. Cambridge. Hunter-Anderson, R. L. (1977): «A theoretical approach to the study of house form». En Binford, L. (Ed.): For theory building in archaeology. New York: 287-315.

Iglesias Gil, J. (Ed. 2000): Arqueología en Juliobriga. Universidad de Cantabria. Santander.

Jiménez Ávila, J. (2005): «Cancho Roano: el proceso de privatización de un espacio ideológico». TP. 62 (2): 105-124.

Kaiser, A. (2001): The Urban Dialogue: An analysis of the use of space in the. Roman city of Empuiries, Spain. BAR Int. Series 901. Archaeopress. Oxford.

Kent, S. (Ed.) (1990): Domestic architecture and the use of space. Cambridge University Press. New York.

Kopytoff, I. (1986): «The cultural biography of things: commoditization as process». En Appaidurai, A. (Ed.): The social life of things. Commodities in cultural perspective. University Press. Cambridge: 64-91.

Laurence, R. (1994): Roman Pompeii: space and society. Routledge. Londres.

Lefebvre, H. (1991): The Production of Space. University Press. Cambridge.

Leroi-Gourham, A. (1984). Simbolos, Artes y Creencias de la Prehistoria. Istmo. Madrid.

Lévi-Strauss, C. (1991): Voz «Maison». En Bonte, P. y Izard, M. (Eds.): Dictionnaire de l'ethnologie et de l'anthropologie. Presses Univiersitaires de France. Paris.

Lévi-Strauss, C. (2000 [1961]): Antropología estructural. Paidós. Barcelona.

Manum, B. Rusten, E. y Benze, P. (2005): «AGRAPH. Software for Drawing and Calculating Space Syntex Graphs». En Van Nees, A: (Ed.): Proceedings of the $5^{\text {th }}$ Space Syntax Symposium, June 2005. Techne Press. Amsterdam: 96-103.

Norberg-Schulz, C. (1963): Intentions in Architecture. Oslo.

Norberg-Schulz, C. (1988): Nightlands: nordic buildings. Mass. Cambridge.

Rapoport, A. (1969): House form and culture. Londres.

Rapoport, A. (1990): The meaning of the built environment: a non-verbal communication approach. University of Arizona. Tucson.

Rapoport, A. (1990): «Systems of activities and systems of settings». En Kent, S. (Ed.): Domestic architecture and the use of space: An interdisciplinary crosscultural study. NDA. University Press. Cambridge: 9-20.

Richter, M. (1955): Espacio y ambiente en la arquitectura moderna. Gustavo Gili. Barcelona.

Sánchez, J. (1998): «La arqueología de la Arquitectura. Aplicación de nuevos modelos de analítica a estructuras de la Alta Andalucía en época ibérica». TP. 55 (2): 89-109.

Saussare, F. (1964 [1916]): Curso de lingüistica general. Ed. Losada. Buenos Aires. Schiffer, M. B. (1985): «Is there a 'Pompeii Premise' in archaeology?». JAR 41 (1): $18-41$.

Schiffer, M. B. (1987): Formation processes of archaeological record. New México University Press. Albuquerque.

Turner, A.; Doxa, M. ;O'Sullivan, D. y Penn, A. (2001) : «From isovists to visibility graphs: a methodology for the analysis of architectural space». Environment and Planning B. 28:103-21.

Vela Cossío, F. (2002): Espacio doméstico y arquitectura del territorio en la prehistoria peninsular: tipología y razón constructiva en la arquitectura celtibérica. Tesis doctoral leída en el Departamento de Prehistoria de la Universidad Complutense. Madrid.

Westgate, R. (2007): «House and society in Classical and Hellenistic Crete: a case study in regional variation». AJA 111: 423-57.

Widlok, T. (1999): «Mapping Spatial and Social Permeability». Current Anthropology. 21 (3): 392-400.

Zarankin, A. y Niro, C. (2007): «La materialización del sadismo. Arqueología de la arquitectura de los centros clandestinos de detención de la Dictadura militar argentina (1976-1983)». En Funari, P. P. y Zarankin, A. (Eds.): Arqueología de la represión y la resistencia en América Latina, 1960-1980. Facultad de Filosofía y Humanidades. Univerisdad de Catamarca: 159-182.

Zevi, B. (1951): Saber ver la arquitectura: ensayo sobre la interpretación espacial de la arquitectura. Trad. J. Bermejo Godoy. Ed. Poseidón. Buenos Aires.

Recibido: 12 de junio de 2009 Aceptado: 7 de septiembre de 2009 